\title{
EL SISTEMA DE CIENCIA Y TECNOLOGÍA DE COREA DEL SUR: ¿UN EJEMPLO DE COLABORACIÓN INTERNACIONAL PARA EUROPA?
}

\section{THE SCIENCE AND TECHNOLOGY SYSTEM OF SOUTH KOREA: IS IT AN EXAMPLE OF INTERNATIONAL COLLABORATION FOR EUROPE?}

Labra Lillo, Romilio (Centro de Genómica Nutricional Agroacuícola) *

Juan, Myrna (Universidad Complutense de Madrid) ${ }^{* *}$

\section{RESUMEN}

Este artículo analiza el sistema de Ciencia y Tecnología (CyT) de Corea Sur, poniendo atención en la colaboración internacional con la Unión Europea (UE), con el fin de ayudar a comprender el desarrollo económico de Corea, obtener recomendaciones e identificar oportunidades de colaboración para Europa. Para ello, se revisa y analiza información disponible en bases de datos, tratados internacionales de colaboración en CyT entre Corea y la UE, así como antecedentes y estudios previos sobre esta temática. Para tales efectos, el estudio se orienta a analizar la estructura y evolución del sistema coreano de CyT y los principales mecanismos de colaboración con la UE y, a la vez, identificar los principales desafíos y oportunidades para incrementar la actividad científica y tecnológica conjunta Corea-UE. Los resultados obtenidos, junto con reconocer el avance del sistema científico y tecnológico de Corea y las vías seguidas para lograrlo, se evidencia que la colaboración Corea-UE ha sido débil, coexistiendo una gran oportunidad de incrementarla gracias a la coincidencia de estrategias y perspectivas de desarrollo. También es claro que parte importante de la CyT que lleva a cabo Corea es el resultado de la actividad privada y en particular de las empresas multinacionales.

Palabras claves: Ciencia, Tecnología, Colaboración, Corea, Unión Europea. JEL: O33.

\section{ABSTRACT}

This study analyses the Science and Technology (S\&T) system of South Korea, putting special attention on the international collaboration with European Union (EU) in order to understand the Korean progress based on science, as well as to identify new opportunities from scientific cooperation between those economies. To do this, we analyse secondary information available in international databases, documentation related to international treaties of S\&T collaboration of Korea with Europe as well as background and previous studies on the Korean S\&T. The purpose of this paper is to analyse the evolution and structure of the Korean S\&T system, the mechanisms of collaboration with EU, compared with United States of America, and to identify the main challenges and opportunities in order to increase scientific activities between Korea and EU. Along with the evidence that S\&T system of Korea evolved positively, despite the fact that Korea-EU cooperation has been weak, findings also note that there are important ways for future collaborative S\&T works due to the

* Chile. rlabra@ucm.es

** Departamento de Economía Aplicada II. Facultad de Economía y negocios. myrnajuan@gmail.com Recibido: Septiembre de 2015. Aceptado: Diciembre de 2017. 
coincidence of strategies and development perspectives. It is also clear multinational companies in Korea are nowadays an important source of S\&T development.

Key words: Science, Technology, Collaboration, R\&D, European Union, Korea. JEL: O33.

\section{INTRODUCCIÓN}

El desarrollo de Corea es considerado como uno de los más destacados ejemplos de crecimiento observados en los últimos 50 años. Con una economía primaria basada en la agricultura y manufacturera tradicional a inicios del siglo pasado, hoy este país exhibe altos niveles de ingreso (World Bank, 2016) y desarrollo (UNDP, 2015). Las causas de este éxito las podemos encontrar en una combinación de factores socioculturales, geoestratégicos, financieros, comerciales, científicos y tecnológicos, concebidos como parte de una economía basada en el conocimiento (Foray, 2002).

En este contexto, el impulso de la ciencia y tecnología ha jugado un rol clave, tanto para la absorción de tecnologías foráneas en una primera etapa, como para la creación a nivel local en una segunda fase, lo que permitió reducir la brecha tecnológica con los países más avanzados, y lograr posicionarse como líder en diversos campos intensivos en conocimiento, tales como electrónica, informática, transporte, entre otros (Aizawam et al., 2007).

Es por ello la relevancia de analizar el rol de la ciencia y tecnología (CyT) en el desarrollo de Corea, mirando en retrospectiva para ilustrar posibles claves que puedan derivarse y ser aplicadas en realidades similares. De esta manera, este trabajo busca analizar comparativamente la colaboración en Ciencia y Tecnología de Corea y dos líderes mundiales, Estados Unidos de América (EUA) y la UE, teniendo en cuenta los elementos socioculturales, económicos e históricos que pudieran condicionarla, de forma tal que ayude a mejorar nuestra comprensión del efecto de la ciencia en el desarrollo de esta nación. Asimismo, se busca identificar oportunidades para que Europa incremente su interacción con Corea, como mecanismo para impulsar la economía comunitaria y enfrentar conjuntamente los grandes desafíos de la sociedad, sobre la base del conocimiento y la innovación (European Commision, 2015). Adicionalmente, se busca caracterizar los principales desafíos y oportunidades que podrían resultar de un incremento de la actividad conjunta en CyT entre Corea y la UE y de esta forma, impulsar el crecimiento de ambos socios.

Este artículo hace precisamente una breve revisión de los aspectos socioculturales e históricos más destacados que han impactado el progreso de Corea y, en particular, la implicación de la CyT en este proceso. Asimismo, se ofrece una visión sobre la conformación de su sistema de innovación, las perspectivas estratégicas y los principales puntos críticos. Finalmente, se realiza una revisión de la colaboración internacional en CyT de Corea, por su efecto determinante en el crecimiento de sus empresas, con un particular análisis del caso de la colaboración con Europa, así como también la posible proyección y oportunidades. De esta forma se intenta responder cuál es el estado actual de la colaboración entre Corea y la UE y cuál ha sido su evolución, así como identificar el camino que ha seguido con EUA, tratando de comprender así cuáles son las diferencias entre ambas colaboraciones en CyT y cuáles han sido sus implicancias en el crecimiento de Corea.

\section{PROPÓSITOS Y METODOLOGÍA}

El desarrollo económico de Corea es considerado como uno de los grandes casos de crecimiento del siglo XX, resultado de una combinación de factores, dentro de los cuales 
destaca el cambio tecnológico, fuertemente sustentado por los flujos provenientes de economías desarrolladas, estimulados por las políticas industriales locales y el interés de sus aliados occidentales por trasformar la economía coreana (Henderson, 1993).

En efecto, la absorción de tecnologías jugó un importante rol, destacando inicialmente aquella proveniente de Estados Unidos, como consecuencia de los planes de desarrollo implementados después de la Segunda Guerra Mundial. Tal como señalan Furman et al.. (2002) en relación al progreso de los países, estas capacidades de incorporar conocimientos foráneos fueron luego acompañadas por procesos endógenos de generación local de conocimientos, emanados en parte de la acumulación de capital intelectual, el que también fue esencial para la identificación, adaptación y adopción de avances logrados en otras latitudes.

Desde una perspectiva más amplia, la apertura de los flujos internacionales de tecnología juega un rol fundamental en la disponibilidad de este tipo de intangibles para una economía emergente como fue la coreana en una primera etapa, pasando desde una posición receptor tecnológico a un intercambiador y proveedor (Athreye y Cantwell, 2007). Según lo señalado por Archibugi y Michie (1995), esta globalización de la tecnología se produce a través de tres principales vías: explotación global de la tecnología; generación global de tecnología y colaboración internacional. De esta forma es posible cerrar la brecha tecnológica gracias a los procesos de colaboración e intercambio y absorción tecnológica, para luego dar paso a una etapa de creación en un ambiente global.

Lee (1997) coincide en señalar que tanto para la absorción como para la creación de tecnologías y conocimientos resulta esencial la colaboración con actores situados más allá de las fronteras nacionales, como una vía para reducir el gap tecnológico y crear capacidades endógenas. La importancia de los procesos de colaboración internacional en CyT para el desarrollo económico de Corea motiva este estudio, fundamentalmente en lo relacionado a los flujos con EUA y la UE, economías líderes en la creación de conocimientos y tecnologías a nivel global. Asimismo, Corea se ha convertido en un importante polo de producción tecnológica y cuenta además con un sistema educativo exitoso (OECD, 2015) que le proporciona una fuente de capital humano altamente cualificado para emprender actividades enfocadas a la innovación. Por ello, Corea se ha transformado en un interesante polo de atracción de la inversión extranjera no sólo por su situación económica, sino, sobre todo, por sus capacidades endógenas científicas y tecnológicas, principalmente en aquellas industrias más sofisticadas (CNUCED, 2015). De esta forma, las Empresas Multinacionales (EMN) se han convertido en un importante impulsor de la CyT local, promoviendo con ello la innovación y el crecimiento (Lee, 2006). Así también, otras vías de difusión internacional de tecnologías siguen siendo relevante para Corea y sus socios, pero fundamentalmente con sus vecinos en Asia y su histórico aliado estratégico: EUA, y no con otros bloques como la UE.

En efecto, mientras que la cooperación en CyT de Corea con EUA es amplia y reconocida, con la UE no estaría teniendo la misma importancia, pero podría ser una nueva vía que permitiese seguir impulsando el desarrollo de esta nación y además, favorecer la estrategia de crecimiento inteligente de Europa a través de flujos bidireccionales (European Comission, 2013a). Si bien esta es una gran oportunidad para la UE, los esfuerzos hasta ahora parecen ser insuficientes para lograr resultados efectivos en dicho sentido.

Por ello, el objetivo general de este trabajo es analizar el sistema de CyT de Corea y su colaboración internacional con la UE y de este modo, mejorar nuestra comprensión de su efecto en el desarrollo de esta nación e identificar oportunidades para Europa. Para ello se han definido tres objetivos específicos: (1) Analizar el sistema de CyT de Corea para comprender su efecto en el desarrollo del país (2) Identificar los principales mecanismos de colaboración 
en CyT entre Corea y EUA y la UE y analizar su evolución; y (3) Caracterizar los principales desafíos y oportunidades que podrían resultar de un incremento de la actividad conjunta en CyT entre Corea y la UE. De lo anterior resulta interesante abordar las siguientes preguntas de investigación: ¿Cuál ha sido el proceso de evolución del sistema de CyT de Corea?, ¿Cuáles han sido los principales canales de cooperación en CyT de Corea con EUA y la UE?, ¿Qué elementos destacados se observan durante la evolución de dicha cooperación, que puedan servir de soporte a nuevas políticas? y ¿Qué oportunidades se visualizan para Europa de aumentar la cooperación en CyT en las áreas definidas como estratégicas para este bloque?

Para abordar este propósito se ha llevado a cabo un estudio de caso sobre Corea, analizando información secundaria provista por las bases de datos: WDI del World Bank (2014); OECD (2013, 2014), The Republic of Korea (2015), MoST y KISTEP (2006 y 2007) y UNESCO (2015). Además, se ha utilizado documentación relacionada a los tratados internacionales de colaboración en CyT de Corea con Europa, así como antecedentes y estudios previos disponibles de la CyT coreana.

\section{DESARROLLO CIENTÍFICO Y TECNOLÓGICO EN COREA}

Desde una perspectiva histórica, el espectacular crecimiento de Corea observado después de la Segunda Guerra Mundial, se puede atribuir inicialmente a la explotación del capital y el trabajo, resultado de políticas de estímulo a la inversión empresarial, alcanzando las más altas cifras de toda el área de la OCDE, así como al crecimiento de su población, largas horas de trabajo, el aumento de la participación en la fuerza laboral y los bajos costos de los factores productivos (García-Blanch, 2002). Sin embargo, el mantenimiento de su crecimiento acelerado durante la segunda parte del siglo pasado y su convergencia con los países líderes, ha sido cada vez más dependiente del impulso a la innovación y a procesos endógenos, como respuesta a la caída en las tasas de Inversión Directa Extranjera (IDE) y las limitantes al ingreso de nuevas tecnologías.

Luego de un periodo de alto crecimiento entre 1970 y 1997, con una tasa promedio del 6,4 por 100 anual (PIB per cápita entre 1970 y 1997), la situación cambió y la expansión del PIB se desaceleró, como se puede observar en el gráfico siguiente (gráfico 1). Posterior a la crisis de finales de la década de 1990, Corea mostró una tendencia decreciente en su dinamismo económico, aun cuando su tasa media anual de crecimiento a partir de 1999 fue del 4,1 por 100, una de las más altas en el área de la OCDE (OECD, 1996).

\section{GRÁFICO 1: TASA DE CRECIMIENTO DEL PIB PER CÁPITA ENTRE 1961 Y 2012. COREA DEL SUR}

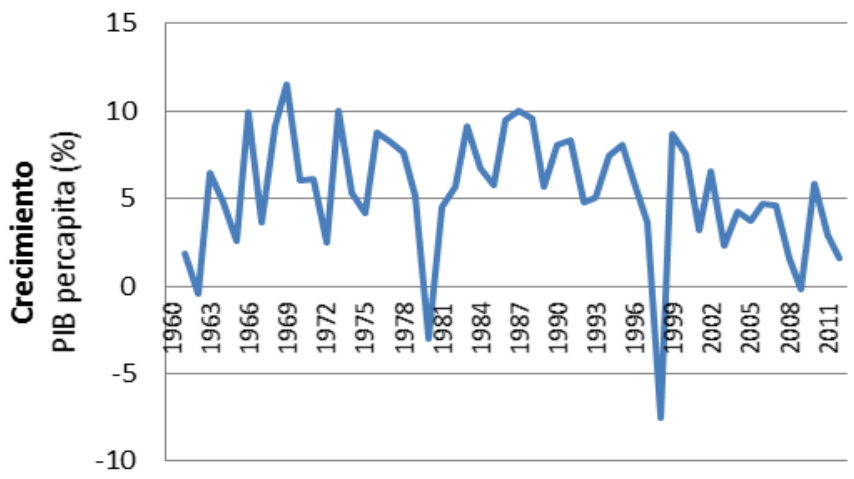

Fuente: World Bank (2014) 
Esta baja en la tasa de crecimiento se debió fundamentalmente a la caída en el consumo privado, la formación fija bruta de capital y la demanda nacional total (Anexo 1). En Corea estas tendencias fueron interpretadas como señales de alerta de un debilitamiento en el crecimiento potencial de la economía. Sin embargo, estos signos de declive se deben poner en perspectiva y analizar más profundamente, ya que detrás de estas cifras, está el fuerte auge de las Tecnologías de Información y Comunicación (TICs) en la década del 2000 y el exagerado aumento del consumo privado asociado a la burbuja inmobiliaria (OECD, 2013). Lo anterior vino a poner de manifiesto que posibles causas estructurales podrían estar afectando el progreso económico de Corea en el largo plazo.

A pesar de esta desaceleración en la última parte del siglo pasado, la economía coreana sigue siendo un ejemplo de crecimiento. Los analistas tienden a señalar que estos resultados positivos pareciesen estar cimentados en tres pilares fundamentales: una política activa de Investigación y Desarrollo (I+D), actividad impulsada tanto desde el sector privado como público; un sistema educativo exitoso con un alto estándar internacional; políticas macro y microeconómicas tendientes a la apertura, estabilidad y desarrollo industrial intensivo en conocimiento (Suh, 2000).

\subsection{Investigación y desarrollo en Corea. Un proceso evolutivo conectado con el crecimiento económico}

Las políticas industriales en Corea han tenido un claro acento en la especialización de determinados sectores, definidos como estratégicos para el desarrollo del país. En una primera etapa (décadas de los 60 y 70) las industrias pesadas y la química recibieron la atención del Estado, así como también se dieron los primeros pasos hacia el desarrollo del sector de las TICs hacia fines de ese período. En la década de los 80 el énfasis fue puesto en la transformación estructural de la economía, expandiéndose hacia industrias intensivas en tecnología, tales como maquinaria sofisticada y electrónica. Esta etapa marcó una clara orientación hacia la consolidación de las capacidades locales de generación de tecnologías tanto en centros públicos como en las empresas, las cuales comenzaron a establecer sus propios laboratorios para cubrir sus necesidades tecnológicas (Eckert, 1990; MoST, 2013) A partir de ese momento, quedaron sentadas la bases del liderazgo industrial y tecnológico que hoy en día disfruta Corea en diversos ámbitos, fundamentalmente el naviero, electrónica, automotriz y petroquímico (ICEX, 2013).

En términos de I+D, Corea ha experimentado un fuerte incremento del gasto y en el número de investigadores dedicados a esta labor, los que casi se han triplicado desde 1997 (cuadro 1). En términos equivalentes a investigadores de tiempo completo (FTE), existen aproximadamente 200,000 investigadores, lo que se traduce en 12,8 investigadores por cada 1,000 empleados totales.

En términos comparados, estas cifras muestran que Corea avanzó más rápidamente en la acumulación de capital humano dedicado a labores de I+D, superando largamente en la actualidad a la dotación de investigadores de EUA, y a la media de la OECD y UE.

Como podemos observar en el cuadro 2, del total de investigadores en Corea, alrededor de 96.000 fueron empleados por las universidades en 2011, lo que representa un poco más del 25por 100, y alrededor de cuatro veces más de los que trabajan en los Government Research Institutes (GRIs), a pesar de los altos gastos de investigación que estos últimos realizan. Además se observa una tendencia a disminuir la participación de investigadores en la academia e incrementar en la empresa privada, lo que demuestra un cambio en el patrón de desarrollo de la I+D del país (MoST y KISTEP, 2004). 


\begin{tabular}{|c|c|c|c|c|c|}
\hline \multicolumn{6}{|c|}{$\begin{array}{c}\text { CUADRO 1: NÚMERO DE PROFESIONALES DEDICADOS A } \\
\text { INVESTIGACIÓN Y DESARROLLO }\end{array}$} \\
\hline \multirow[b]{2}{*}{ Año } & \multicolumn{2}{|c|}{ Corea } & \multirow{2}{*}{$\begin{array}{l}\text { OECD } \\
\text { Total por } 1000 \\
\text { empleados }\end{array}$} & \multirow{2}{*}{$\begin{array}{c}\text { EUA } \\
\text { Total por } 1000 \\
\text { empleados }\end{array}$} & \multirow{2}{*}{$\begin{array}{l}\text { UE } \\
\text { Total por } 1000 \\
\text { empleados } \\
\end{array}$} \\
\hline & $\begin{array}{l}\text { Número total de } \\
\text { empleados en I+D }\end{array}$ & $\begin{array}{l}\text { Total por } 1000 \\
\text { empleados }\end{array}$ & & & \\
\hline 1997 & 138.438 & 48,422 & 56,926 & 66,684 & 48,606 \\
\hline 1998 & 129.767 & 46,456 & 58,730 & 69,985 & 49,699 \\
\hline 1999 & 134.568 & 49,425 & 60,131 & 70,976 & 51,125 \\
\hline 2000 & 159.973 & 51,270 & 60,822 & 70,645 & 52,374 \\
\hline 2001 & 178.937 & 63,245 & 62,871 & 72,784 & 53,947 \\
\hline 2002 & 189.888 & 64,068 & 63,946 & 75,446 & 56,108 \\
\hline 2003 & 198.171 & 68,390 & 67,180 & 80,398 & 57,970 \\
\hline 2004 & 209.979 & 69,255 & 67,406 & 78,061 & 59,973 \\
\hline 2005 & 234.702 & 78,672 & 69,055 & 76,473 & 62,376 \\
\hline 2006 & 256.598 & 86,386 & 69,938 & 77,052 & 63,472 \\
\hline 2007 & 289.098 & 94,708 & 69,800 & 76,439 & 63,872 \\
\hline 2008 & 300.050 & 100,154 & 71,511 & 80,669 & 66,015 \\
\hline 2009 & 323.175 & 103,838 & 74,860 & 87,984 & 68,577 \\
\hline 2010 & 345.912 & 110,840 & 74,997 & 84,752 & 71,034 \\
\hline 2011 & 375.176 & 119,163 & 76,852 & 88,122 & 71,947 \\
\hline 2012 & 401.724 & 127,869 & 77,830 & 87,396 & 74,392 \\
\hline 2013 & 410.333 & 128,396 & $:::$ & $:::$ & 76,825 \\
\hline 2014 & 437.447 & 13,495 & $:::$ & $:::$ & 7,776 \\
\hline
\end{tabular}

\section{GRÁFICO 2: GASTO EN I+D DE LA UE, COREA Y EUA ENTRE 1996 Y 2011}



$$
\text { - - -UE —Corea - - USA }
$$

Fuente: elaboración propia a partir de datos del World Bank (2014) 


\begin{tabular}{|c|c|c|c|c|c|c|c|c|c|c|c|c|c|c|c|}
\hline \multicolumn{16}{|c|}{ CUADRO 2: GASTOS EN INVESTIGACIÓN Y DESARROLLO (GERD) EN COREA } \\
\hline Indicador/Año & 1997 & 1998 & 1999 & 2000 & 2001 & 2002 & 2003 & 2004 & 2005 & 2006 & 2007 & 2008 & 2009 & 2010 & 2011 \\
\hline $\begin{array}{l}\text { Total de investigadores } \\
\text { (Empleados) }\end{array}$ & 138.438 & 129.767 & 134.568 & 159.973 & 178.937 & 189.888 & 198.171 & 209.979 & 234.702 & 256.598 & 289.098 & 300.050 & 323.175 & 345.912 & 375.176 \\
\hline (\%) del total & $100 \%$ & $100 \%$ & $100 \%$ & $100 \%$ & $100 \%$ & $100 \%$ & $100 \%$ & $100 \%$ & $100 \%$ & $100 \%$ & $100 \%$ & $100 \%$ & $100 \%$ & $100 \%$ & $100 \%$ \\
\hline $\begin{array}{l}\text { Total del gasto bruto en } I+D_{1} \\
\text { (GERD)* }\end{array}$ & 19.745 .884 & 17.359 .249 & 18.273 .401 & 20.213 .1932 & 22.640 .9002 & 23.585 .879 & 25.066 .8632 & 28.305.232 & 30.618 .3263 & 34.711 .9883 & 38.923 .4804 & 11.685.344 & 4.311.229 & 49.447 .736 & 55.402 .128 \\
\hline (\%) del total & $100 \%$ & $100 \%$ & $100 \%$ & $100 \%$ & $100 \%$ & $100 \%$ & $100 \%$ & $100 \%$ & $99 \%$ & $100 \%$ & $100 \%$ & $100 \%$ & $100 \%$ & $100 \%$ & $100 \%$ \\
\hline $\begin{array}{l}\text { GERD por investigador, Empleados } \\
(*)\end{array}$ & 142,60 & 133,80 & 135,80 & 126,40 & 126,50 & 124,20 & 126,50 & 134,80 & 130,50 & 135,30 & 134,60 & 138,90 & 137,10 & 142,90 & 147,70 \\
\hline $\begin{array}{l}\text { Investigadores (Empleados) - } \\
\text { Privada sin fines de lucro }\end{array}$ & 1.493 & 1.112 & 1.519 & 1.422 & 1.186 & 1.627 & 1.759 & 1.703 & 2.036 & 2.181 & 2.883 & 3.673 & 3.711 & 4.217 & 5.090 \\
\hline (\%) of total & $1,08 \%$ & $0,86 \%$ & $1,13 \%$ & $0,89 \%$ & $0,66 \%$ & $0,86 \%$ & $0,89 \%$ & $0,81 \%$ & $0,87 \%$ & $0,85 \%$ & $1,00 \%$ & $1,22 \%$ & $1,15 \%$ & $1,22 \%$ & $1,36 \%$ \\
\hline $\begin{array}{l}\text { GERD - Financiado por entidades } \\
\text { privadas sin fines de lucro }(*)\end{array}$ & 178.858 & 68.496 & 225.239 & 67.330 & 88.294 & 93.024 & 92.632 & 110.609 & 122.494 & 121.265 & 134.249 & 164.958 & 183.101 & 188.076 & 243.952 \\
\hline (\%) del total & $0,91 \%$ & $0,39 \%$ & $1,23 \%$ & $0,33 \%$ & $0,39 \%$ & $0,39 \%$ & $0,37 \%$ & $0,39 \%$ & $0,40 \%$ & $0,35 \%$ & $0,34 \%$ & $0,40 \%$ & $0,41 \%$ & $0,38 \%$ & $0,44 \%$ \\
\hline $\begin{array}{l}\text { Investigadores (Empleados) - En } \\
\text { universidades }\end{array}$ & 48.588 & 51.162 & 50.151 & 51.727 & 53.717 & 57.634 & 59.746 & 61.388 & 64.895 & 65.923 & 83.123 & 82.077 & 88.554 & 93.509 & 95.750 \\
\hline (\%) del total & $35,10 \%$ & $39,43 \%$ & $37,27 \%$ & $32,33 \%$ & $30,02 \%$ & $30,35 \%$ & $30,15 \%$ & $29,24 \%$ & $27,65 \%$ & $25,69 \%$ & $28,75 \%$ & $27,35 \%$ & $27,40 \%$ & $27,03 \%$ & $25,52 \%$ \\
\hline $\begin{array}{l}\text { GERD } \quad \text { Financiado } \\
\text { universidades }(*)\end{array}$ & 715.412 & 775.931 & 703.124 & 664.508 & 390.670 & 373.671 & 337.611 & 288.443 & 277.334 & 288.573 & 383.078 & 419.449 & 399.133 & 421.315 & 399.093 \\
\hline (\%) del total & $3,62 \%$ & $4,47 \%$ & $3,85 \%$ & $3,29 \%$ & $1,73 \%$ & $1,58 \%$ & $1,35 \%$ & $1,02 \%$ & $0,91 \%$ & $0,83 \%$ & $0,98 \%$ & $1,01 \%$ & $0,90 \%$ & $0,85 \%$ & $0,72 \%$ \\
\hline $\begin{array}{l}\text { Investigadores (Empleados) - } \\
\text { Gobiernos }\end{array}$ & 13.692 & 11.475 & 12.467 & 12.491 & 12.735 & 12.467 & 12.636 & 12.588 & 13.465 & 14.590 & 17.459 & 17.277 & 20.607 & 22.018 & 23.710 \\
\hline (\%) del total & $9,89 \%$ & $8,84 \%$ & $9,26 \%$ & $7,81 \%$ & $7,12 \%$ & $6,57 \%$ & 6,38\% & $5,99 \%$ & $5,74 \%$ & $5,69 \%$ & $6,04 \%$ & $5,76 \%$ & $6,38 \%$ & $6,37 \%$ & 6,32\% \\
\hline $\begin{array}{l}\text { GERD - Financiado por el } \\
\text { gobierno }\left(^{*}\right)\end{array}$ & 4.525 .671 & 4.501.339 & 4.550 .354 & 4.839.216 & 5.650 .509 & 5.986 .148 & 5.979 .830 & 6.549 .345 & 7.049 .806 & 8.008.794 & 9.651 .4251 & 10.591 .82612 & 2.139.136 & 13.225 .685 & 13.796 .786 \\
\hline (\%) del total & $22,92 \%$ & $25,93 \%$ & $24,90 \%$ & $23,94 \%$ & $24,96 \%$ & $25,38 \%$ & $23,86 \%$ & $23,14 \%$ & $23,02 \%$ & $23,07 \%$ & $24,80 \%$ & $25,41 \%$ & $27,40 \%$ & $26,75 \%$ & $24,90 \%$ \\
\hline $\begin{array}{l}\text { Investigadores (Empleados) } \\
\text { Empresas }\end{array}$ & 74.665 & 66.018 & 70.431 & 94.333 & 111.299 & 118.160 & 124.030 & 134.300 & 154.306 & 173.904 & 185.633 & 197.023 & 210.303 & 226.168 & 250.626 \\
\hline (\%) del total & $53,93 \%$ & $50,87 \%$ & $52,34 \%$ & $58,97 \%$ & $62,20 \%$ & $62,23 \%$ & $62,59 \%$ & $63,96 \%$ & $65,75 \%$ & $67,77 \%$ & $64,21 \%$ & $65,66 \%$ & $65,07 \%$ & $65,38 \%$ & $66,80 \%$ \\
\hline $\begin{array}{l}\text { GERD - Financiado por Empresas } 1 \\
(*)\end{array}$ & 14.306.831 & 12.000 .571 & 12.783 .431 & 14.630 .1051 & 16.405 .0111 & 17.029.195 & 18.553 .1272 & 21.218.502 & 22.951 .3912 & 26.188 .9242 & 28.668 .0473 & 30.380 .88031 & 1.498.361 & 35.505 .561 & 40.838 .114 \\
\hline (\%) del total & $72,45 \%$ & $69,13 \%$ & $69,96 \%$ & $72,38 \%$ & $72,46 \%$ & $72,20 \%$ & $74,01 \%$ & $74,96 \%$ & $74,96 \%$ & $75,45 \%$ & $73,65 \%$ & $72,88 \%$ & $71,08 \%$ & $71,80 \%$ & $73,71 \%$ \\
\hline
\end{tabular}


Por su parte, los GRIs emplean al 70 por 100 de los doctores en Corea, sin embargo, representan sólo alrededor del 10 por 100 del gasto en investigación. Esto es, en parte, debido a que en la mayoría de las universidades coreanas, las instalaciones de $\mathrm{I}+\mathrm{D}$ y sus equipos de investigación no están bien organizados y tienen menor productividad que en los radicados en los GRIs (UNESCO, 2015).

También cabe destacar la gran importancia que tiene el sector privado en la financiación de la I+D, representado alrededor del 70 por 100 del total del gasto en $\mathrm{I}+\mathrm{D}$, porcentaje que se mantienen estable a lo largo del período analizado. En efecto, el sector privado es el mayor inversor en $\mathrm{I}+\mathrm{D}$, lo que representa alrededor de tres cuartas partes de los gastos de Corea, pero esto no ha sido así durante los últimos 25 años.

Por su parte, la tasa de crecimiento anual del gasto privado en I+D es de alrededor de dos veces el promedio de la OCDE, lo cual es indicativo de la irrupción de Corea como líder en tecnología industrial, especialmente en TICs, sector de los automóviles, construcción naval y en la industria del acero (OECD, 2009a). Lo anterior refleja el dinamismo que ha alcanzado el sector privado como motor de la innovación en Corea, el que por la estructura del tejido empresarial surcoreano tiene un fuerte componente de Empresas Multinacionales. Así entonces, se puede entender que gran parte de la CyT es impulsada por este tipo de compañías, en plena concordancia con lo planteado por Cuervo-Cazurra (2007) y la teoría de la internacionalización de la CyT de las empresas y su efecto en los sistemas de innovación local.

A nivel del sector público, el mayor aporte estatal se ha dirigido a los GRIs, mientras que el gobierno coreano espera movilizar la investigación hacia las universidades, puesto que son consideradas como un punto clave para que Corea logre una mejor posición con respecto a las principales economías. Sin embargo, la falta histórica de inversión en investigadores en las universidades, supone un gran obstáculo para el desarrollo de capacidades.

En términos relativos, Corea presenta un gasto en I+D superior a EUA y la UE, llegando a valores del 3,7 por 100 del PIB, situación que ha evolucionado positivamente en los últimas tres décadas. Es claro que valores sobre el 3 por 100 son un excelente indicador de la importancia que se le otorga a la $\mathrm{I}+\mathrm{D}$ en Corea, modelo que pudiera ser de utilidad para incentivar la actividad científica en los países más rezagados de la UE (OECD, 2009b).

Todo este esfuerzo ha llevado a Corea a situarse en la frontera tecnológica. En los últimos años, ha alcanzado a los países más avanzados tecnológicamente. Lo anterior ha implicado nuevos desafíos, destacando la necesidad de impulsar investigaciones más fundamentales. Esto ha dado lugar a un gradual cambio al tipo de la I+D que realiza. Se ha producido un aumento en la investigación básica y desarrollo experimental, al tiempo que el gasto en investigación aplicada se ha reducido (OECD, 2009a).

Este hecho queda de manifiesto si se analiza la producción científica (artículos científicos) y tecnológica (medido a través de las patentes) (gráficos 3 y 4), donde resalta esta última, por sobre la productividad en ciencia de los investigadores.

Es importante notar que el bajo número de artículos científicos también es explicado por el hecho de que el sector privado lleva a cabo la mayor parte de la I+D, el cual percibe pocos incentivos para abrir el conocimiento a terceros actores, en contraposición a la protección de las invenciones a través de patentes. Lo anterior queda reflejado al observar las patentes concedidas totales (gráfico 4), donde Corea muestra un gran desempeño con un marcado ascenso a partir de la década de 1990, sobrepasando a EUA y la UE. En este sentido, posibles recomendaciones podrían derivarse a partir del caso coreano para la UE, con el fin de mejorar los resultados de la actividad de I+D con mayor orientación a la industria. 


\section{GRÁFICO 3: PRODUCTIVIDAD CIENTÍFICA (ARTÍCULOS CIENTÍFICOS POR CADA 1000 INVESTIGADORES) DE COREA, EUA Y LA UE}

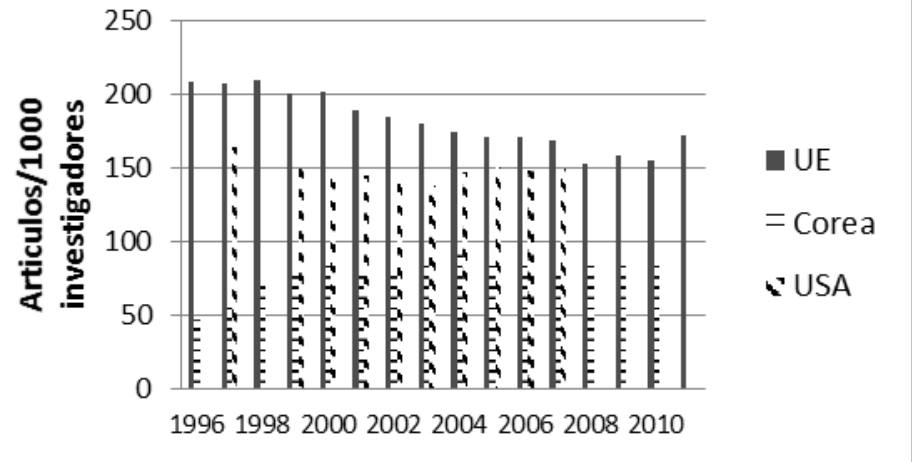

Fuente: Elaboración propia a partir de datos de World Bank (2014)

\section{GRÁFICO 4: PATENTES (CONCESIÓN DE PATENTES MILLÓN DE HABITANTES) DE COREA, EUA Y LA UE}

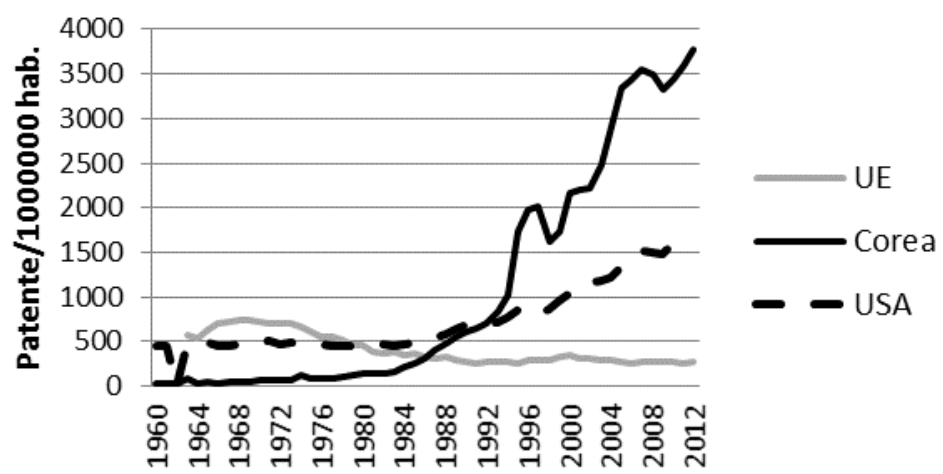

Fuente: Elaboración propia a partir de datos de World Bank (2014)

La ambiciosa estrategia coreana en CyT, que busca seguir siendo el soporte fundamental de su desarrollo, contempla cinco objetivos (Foreign and Commonwealth Office, 2013):

- Incremento de la inversión en I+D maximizando la eficiencia; desarrollo estratégico de tecnologías con 30 prioridades y 120 estrategias para cubrir los campos de energía, medioambiente, TICs y sanidad. Específicamente se pondrá énfasis en redes inteligentes, CCS, aplicaciones para grandes datos $\mathrm{y}$ medicamentos personalizados.

- Construcción de capacidades creativas de medio y largo plazo, incrementando los fondos para las ciencias básicas e intercambios internacionales.

- Mayor apoyo a las PYMEs y Start-Ups en nuevas industrias

- Brindar un mayor impulso a la generación y comercialización de la propiedad intelectual.

- Creación de nuevos empleos basados en ciencia. 


\subsection{Sistema educativo y la ciencia en Corea}

El sistema educativo coreano es uno de los grandes pilares de su desarrollo industrial. Desde las primeras etapas del desarrollo coreano, las políticas han estado orientadas a la creciente consecución de actividades de investigación y desarrollo, y a la formación de capital humano. Este último aspecto ha ocupado un lugar central, no sólo de las políticas de crecimiento económico, sino también en la promoción y conservación de sus valores y cultura. Facilitado y promovido desde el Estado, las familias impulsan la formación de los niños desde temprana edad, inculcando una alta conciencia sobre la necesidad de la educación hasta el nivel profesional, procurando el acceso a los mejores centros de enseñanza, tanto nacional como internacional.

Los resultados respecto de la calidad de la educación en ese país son asombrosos, lo que le han permitido situarse en los primeros lugares del mundo según la evaluación PISA (OECD, 2013), muy por encima de EUA y la mayoría de los países europeos. Este es un claro ejemplo de la capacidad para formar capital humano avanzado en una economía que tiende cada vez más a generar sus propias tecnologías y reducir la dependencia internacional, una estrategia que además, intenta mantener un fuerte vínculo con la sociedad y la industria (OECD, 2006).

Estos logros son también, en parte, consecuencia de una dedicación preferente de las universidades a la actividad académica sobre la científica. Por ello, el Estado impulsó la creación de los Institutos de Investigación del Gobierno (GRIs), cuya misión fue acelerar la adopción y adaptación de tecnologías por parte de las empresas coreanas. Esto permitió que las universidades coreanas se dedicasen de forma casi exclusiva a la enseñanza, dejando la I+D en manos de las empresas, con apoyo de los Institutos de investigación, quienes al día de hoy son los mayores inversores y ejecutores de la I+D en Corea (UNESCO, 2015). Durante los últimos años el gobierno ha hecho un esfuerzo para fortalecer las capacidades de investigación en las universidades, pero este es un proyecto a largo plazo y no exento de dificultades.

A pesar de los éxitos en la educación, los estudiantes coreanos para conseguir puntuaciones altas en el examen de ingreso a las universidades de élite promueven una educación que enfatiza el razonamiento científico, dejando de lado la capacidad de resolver y tomar decisiones en situaciones cotidianas relacionadas con la ciencia y la tecnología, lo que conlleva una debilidad, pues los estudiantes deben ser capaces de resolver problemas donde no existen soluciones basadas en reglas claras y, además, necesitan comunicar ideas científicas complejas de forma explícita y persuasiva, para lo cual no están tan bien preparados (OECD, 2007).

Los antecedentes indican que aunque la educación en Corea intenta adaptarse, el aprendizaje sigue estando basado en la memoria y deja poco espacio al pensamiento creativo y el espíritu de exploración, que son esenciales para la CyT (Bouteiller y Fouquin, 2001).

\subsection{Organización pública del sistema de ciencia y tecnología}

Después de un largo período con un Estado de importantes dimensiones y poco ágil, a partir de 2008 el gobierno de Corea impulsó una reestructuración institucional enfocada a reducir el tamaño del gobierno, fusionándose la mayor parte del Ministerio de Ciencia y Tecnología (MoST) y el Ministerio de Educación y del Desarrollo de Recursos Humanos (MoE) en el Ministerio de Educación, Ciencia y Tecnología (MEST), mientras que el Ministerio de Comercio Industria y Energía (MoCIE) y el Ministerio de Información y 
Comunicaciones (MIC) se han reunido en el Ministerio de Economía del Conocimiento (MKE), tal y como aparece en la figura 1. Aun cuando estos cambios buscaban una mayor eficiencia e impulsar una nueva etapa de desarrollo, con un mayor énfasis en la internacionalización y en los temas energéticos, es claro que se dio un importante paso para fortalecer la institucionalidad relacionada a la innovación y formación de capital humano, fundamentalmente en el desarrollo de capacidades de absorción y transformación industrial.

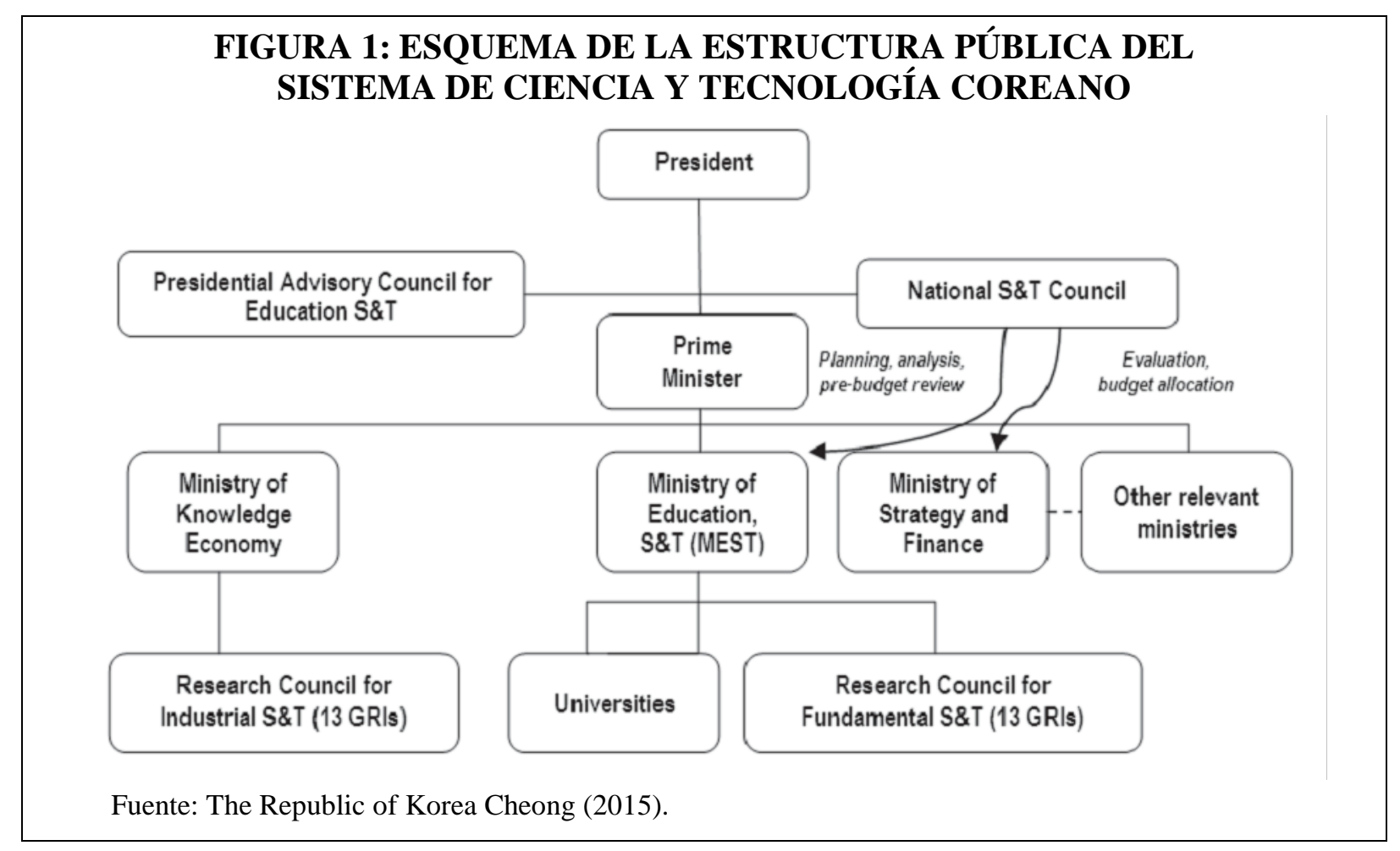

Así, el MoST fue el ministerio más importante para la formulación de políticas de CyT, siendo responsable de planificar, dirigir y evaluar todas las acciones de CyT en Corea y de formular las políticas y programas de apoyo al desarrollo nacional. Por su parte, el MoE fue el responsable de la formulación, planificación y coordinación de las políticas educativas.

El MEST, por su parte, es el encargado en la actualidad de la educación y del sistema de ciencia y tecnología. Sin embargo, existen cruces con el Ministerio de Educación, quien se ocupa de la política de investigación académica (MEST, 2008).

Otro importante ministerio creado luego de la reestructuración de fines de la década pasada, es el Ministerio de Economía del Conocimiento (MKE), el cual es resultante de la fusión del antiguo Ministerio de Comercio, Industria y Energía (MoCIE) y el Ministerio de Información y Comunicaciones (MIC), incorporándose también algunos elementos de Ministerio de Ciencia y Tecnología (MoST). Este nuevo ministerio, tiene un claro énfasis en el impulso de una industria basada en el conocimiento y en el fortalecimiento de las diversas fuentes de energía, todo esto bajo una intensa colaboración internacional. 


\subsection{Del Plan de CyT 2003-2007 a la Visión de Largo plazo para el desarrollo de la CyT}

El plan de CyT se revisó con la entrada del gobierno de Roh Moo-hyun en febrero de 2003. En comparación con el plan original básico establecido un año antes, el plan revisado expone más ampliamente el papel y la condición de la CyT, tanto desde una perspectiva nacional como comunitaria. Antes, la CyT podría ser significativa sólo cuando contribuía al desarrollo y la competitividad de la nación. Sin embargo, se introdujo una nueva visión, con lo cual la CyT fue vista desde una perspectiva más amplia. Aun cuando el objetivo central sigue siendo el mismo, hay una nueva mirada del rol del Estado y se incorpora con más fuerza elementos relativos a la competitividad (Park, 2004), que se ha reajustado al alza para convertirse en una de las 8 mejores del mundo en el 2007. Junto a ello, se intensificó la internacionalización del sistema de CyT, el que desde sus inicios había visto cómo la relación con terceros países podía incrementar las oportunidades de desarrollo a partir de la captura de tecnologías foráneas (Hong et al., 2012).

La dirección de la política del Plan Básico revisado es el siguiente:

- Avanzar en el sistema nacional de innovación CyT.

- Seleccionar y centrarse en el futuro estratégico de las áreas de CyT.

- Reforzar futuros motores de crecimiento.

- Sistematizar la capacidad regional de innovación,

- Crear nuevos trabajos que coincidan con las demandas de una sociedad basada en el conocimiento.

- Ampliar la participación de la gente y difundir la cultura CyT,

Las principales indicaciones para el desarrollo de la CyT establecidos en la Visión 2025 incluyen:

- Desplazar el sistema nacional de innovación liderada por el sector privado a otro impulsado por el gobierno.

- Mejorar la eficiencia de las inversiones nacionales de I+D.

- Adaptar el sistema de I+D para los estándares mundiales.

- Cumplir con los desafíos y cosechar las oportunidades que presentan las nuevas tecnologías.

Este es un esfuerzo por hacer realidad la visión para el año 2025 que el gobierno de Corea ha puesto en marcha por medio del 21 Century Frontier S\&D Programm y la promulgación de la Ley Marco de Ciencia y Tecnología en 1999. Sobre la base de esta ley, el Gobierno formuló el Plan de Ciencia y Tecnología de Cinco Años y así como una Hoja de Ruta Nacional de Tecnología (Lim, 2000).

\subsection{Sector privado y la innovación}

El sector privado en Corea es en la actualidad el gran motor de la I+D. Además de ser el principal agente que financia la $\mathrm{I}+\mathrm{D}$, es también un importante ejecutor, lo que ha permitido sustentar el desarrollo industrial de alta y media tecnología. Lo anterior se ha visto favorecido por un entorno favorable, que incentiva la inversión en este tipo de actividades.

El gobierno coreano ofrece créditos fiscales a las empresas que llevan a cabo I+D (Ley de Desarrollo Industrial, promulgada en 1985), y más del 50 por 100 del gasto gubernamental en $\mathrm{I}+\mathrm{D}$ se dirige al desarrollo industrial. Además, el gobierno también está activo en la 
implementación de las políticas y programas que se ocupan de una amplia gama de cuestiones relacionadas con la innovación en el sector privado, dentro de ellas están:

- El riesgo de la financiación.

- El desarrollo de habilidades.

- La colaboración entre la industria y la académica

- El fortalecimiento de los clústers.

Las actuales políticas públicas de innovación empresarial, procuran reducir la brecha entre los chaebol (estructura empresarial familiar coreana) y las PYMEs. En este sentido, esta nueva visión enfatiza la importancia de las PYMEs como un nuevo eje para el desarrollo, intentando que la experiencia y éxitos alcanzados por los chaebol se difundan, así como resolver los obstáculos al progreso que genera este tipo de estructuras empresariales tan características en Corea. Los datos muestran que la innovación en las PYMEs está aún muy lejos de lo que alcanzan los chaebol, por ello el gobierno hace esfuerzos por apoyos focalizados para facilitar el acceso a tecnología y a financiación de la I+D en este segmento.

Asimismo, los chaebol también están jugando un importante rol en el desarrollo de las PYMEs, pues estás deben mejorar su condición de proveedores, lo que a su vez ha incrementado las capacidades de $\mathrm{I}+\mathrm{D}$, con el fin de satisfacer la creciente demanda tecnológica de los chaebol. Este hecho ha ayudado además a reducir la dependencia a un único chaebol, como resultado de sus mayores competencias y ampliación de sus redes.

Aun cuando las empresas nacionales continúan ocupando parte importante de la escena productiva local, en la última década Corea ha desarrollado una gran capacidad para atraer inversión extranjera directa (IED). Actualmente esta nación se ubica en el séptimo lugar dentro de los países más atractivos de Asia del este y sureste para los inversionistas, como consecuencia del gran desarrollo económico y la especialización en TICs (CNUCED, 2015). De esta forma, un importante motor de la innovación en el sector de las TICs lo constituye las EMN extranjeras, las que sumadas a la manufactureras locales conforman una estructura integrada muy activa en términos de innovación.

\section{COLABORACIÓN INTERNACIONAL EN CIENCIA Y TECNOLOGÍA: COREA-UE}

Desde hace más de tres décadas Corea definió como piedra angular de su desarrollo a la Ciencia y Tecnología y, con este propósito impulsó una serie de estrategias, leyes y programas de trabajo que permitiesen el crecimiento de las capacidades locales para absorber y adoptar tecnologías foráneas y posteriormente desarrollar las propias (MoST, 2013), visión que fue reforzada con la creación del Ministerio de Ciencia y tecnología.

Junto al desarrollo económico, sus empresas se fueron internacionalizando cada vez más, lo que se unió a las políticas de apertura implementadas por el país, que incentivaban tanto las exportaciones de bienes como la inversión extranjera directa y la importación de tecnologías. En este escenario, el sector privado tuvo un rol protagónico, destacando la labor desempeñada por los chaebol como agentes de la trasformación industrial y apertura económica, con un fuerte apoyo del sector público. Hacia fines del siglo XX y luego de la denominada Crisis Asiática (fines de la década de los 90`s), que afectó fuertemente a Corea, el énfasis de las políticas relacionadas a CyT se orientaron a mejorar la eficiencia de los recursos disponibles e incrementar el desempeño de las competencias adquiridas y del capital humano acumulado, con el fin último de aumentar la competitividad industrial. Esto dio 
también un nuevo impulso a la internacionalización de las empresas, la inversión extranjera y a la colaboración internacional en CyT.

Las políticas de CyT, de igual forma que la economía, no fueron geográficamente cerradas sino que por el contrario, buscaron mecanismos para la absorción e integración de los avances logrados más allá de sus fronteras, así como también la colaboración horizontal. Inicialmente la importación de equipos actuó como vía de entrada de tecnologías al país, lo que fue posible no sólo gracias a la apertura comercial, sino también a las adecuadas capacidades locales para adaptar, imitar y modificar dichas tecnologías, traduciéndose en un aprendizaje. Lo anterior fue acompañado por la formación de profesionales en el extranjero, fundamentalmente en Estados Unidos, Europa y Japón. En el ámbito internacional, esta etapa de captura de tecnologías y desarrollo de capacidades antecedió a otra que se caracterizó por la conducción de procesos más horizontales enfocados a promover la integración, dando pasos hacia el trabajo conjunto y el establecimiento de convenios de colaboración científicotécnico con otras naciones.

En la actualidad, la colaboración internacional en CyT de Corea es conducida y soportada en una serie de acuerdos firmados con diversos países ${ }^{1}$ (Korean National Tax Services), los cuales definen las vías de acción y las prioridades. En este sentido destacan los desarrollos conjuntos e intercambios entre Corea y sus vecinos, China y Japón, así como también con EUA, mientras que con la UE existe una menor interacción.

Las herramientas más frecuentemente empleadas para lograr dichos propósitos corresponden al desarrollo conjunto de proyectos de investigación, flujos de conocimientos y la movilidad de académicos y alumnos. Unido a lo anterior, están los canales propios del sector privado, por medio de la participación en proyectos de $\mathrm{I}+\mathrm{D}$ para la creación de tecnologías con socios situados en cualquier parte del mundo y por medio de los intercambios tecnológicos y de know how, que se generan fundamentalmente a través de las empresas multinacionales.

\subsection{La visión estratégica de la colaboración internacional en CyT de Corea}

Hace más de 40 años Corea estableció claras políticas de desarrollo industrial, las que convergieron hacia el impulso de sectores de alta tecnología, en un ejemplo de transformación industrial. A partir de las prioridades fijadas en aquellos momentos, la estrategia de colaboración internacional se enfocó hacia el fortalecimiento de dichas industrias intensivas en conocimiento, a las cuales se han unido, en los últimos años, los sectores medioambientales y energéticos. Adicionalmente, Corea ha incluido en algunos acuerdos internacionales de cooperación científica y tecnológica más recientes, al sector agrícola, debido al alto impacto social que tiene esta industria en Corea.

Para implementar su estrategia de CyT, Corea se orientó al desarrollo de proyectos de I+D en conjunto con actores internacionales y a la movilidad de investigadores fuera de sus fronteras, con el fin de capturar competencias foráneas. Capítulo aparte ha sido la formación profesional (educación terciaria) de coreanos en el extranjero. Al respecto, un gran número de

\footnotetext{
${ }^{1}$ Albania, Argelia, Australia, Austria, Azerbaiyán, Bahréin, Bangladesh, Bielorrusia, Bélgica, Brasil, Bulgaria, Canadá, Chile, China, Colombia, Croacia, República Checa, Dinamarca, Ecuador, Egipto, Estonia, Fiyi, Finlandia, Francia, Alemania, Grecia, Hungría, Islandia, India, Indonesia, Irán, Irlanda, Israel, Italia, Japón, Jordania, Kazajstán, Kuwait, Kirguistán, Laos, Letonia, Lituania, Luxemburgo, Malasia, Malta, México, Mongolia, Moroco, Myanmar, Nepal, Países Bajos, Nueva Zelandia, Noruega, Omán, Pakistán, Panamá, Papúa Nueva Guinea, Perú, Filipinas, Polonia, Portugal, Qatar, Rumania, Rusia, Arabia Saudita, Singapur, Eslovaquia, Eslovenia, Sudáfrica, España, Sri Lanka, Suecia, Suiza, Tailandia, Túnez, Turquía, Emiratos Árabes Unidos, EUA, Ucrania, Reino Unido, Uruguay, Uzbekistán, Venezuela, Vietnam.
} 
estudiantes adquirieron competencias fuera de sus fronteras (gráfico 5), destacando la presencia de estudiantes coreanos en Estados Unidos, Alemania, Reino Unido y Japón, los que en su conjunto conforman algo más del 3 por 100 de todos los estudiantes coreanos (OECD, 2013). La literatura señala también, que China es y ha sido otro destino seleccionado por una parte importante de los estudiantes coreanos que deciden emigrar para adquirir conocimientos, sin embargo las estadísticas internacionales no recogen apropiadamente esta información.

Claramente, Estados Unidos es el país que más atrae estudiantes coreanos, alcanzando alrededor del 50 por 100 del total de los que emigran para recibir formación terciaria. Dentro de las razones que explican este hecho, están la alta calidad y variedad de oferta educacional que ofrece ese país, principalmente en áreas vinculadas a la tecnología, uno de los principales objetivos de Corea y, los lazos comerciales y geoestratégicos existentes entre ambos países nacidos luego de la Segunda Guerra Mundial, los cuales facilitan y estimulan la concurrencia de estudiantes a dicho país. Esta situación, además de construir nuevas competencias para Corea, ha ido generando inevitablemente cambios sociales al interior del país, dando lugar a nuevos perfiles de identidad en la sociedad coreana.

En segundo lugar, dentro de los destinos para la formación terciaria de estudiantes coreanos, está Japón. Lo anterior está relacionado a la cercanía geográfica, lazos socioculturales y al alto nivel tecnológico de sus universidades y centros de I+D en áreas de interés para Corea. Más atrás se encuentran los países europeos, destacando sólo el Reino Unido y Alemania (gráfico 5).

\section{GRÁFICO 5. ESTUDIANTES COREANOS EN EL EXTRANJERO (PRINCIPALES DESTINOS) EN PROGRAMAS DE EDUCACIÓN TERCIARIA. INCLUYE ESTUDIANTES NO RESIDENTES Y NO CIUDADANOS DE LOS PAÍSES DE DESTINO}
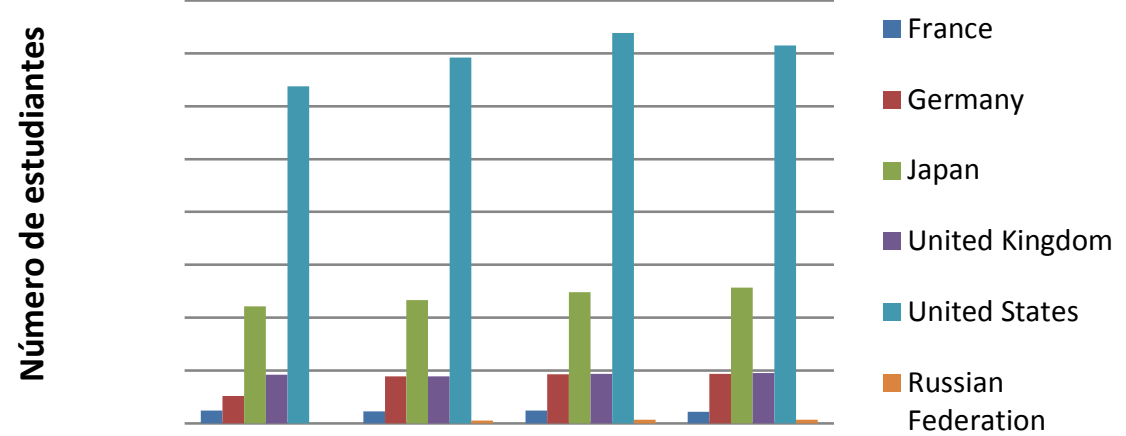

Fuente: Elaboración propia a partir de datos de la OECD (2013)

De lo anterior se desprende que los estudiantes que salen de Corea, lo hacen fundamentalmente atraídos por Estados Unidos, lo que ha servido para ir conformando redes que sustentan nuevos procesos de colaboración científica (proyectos conjuntos, redes de investigación, etc.) entre estos países, mientras que la interacción con Europa sigue siendo débil. 
La estrategia seguida por Corea en este sentido ha sido facilitar los procesos de formación en el extranjero en áreas de relevancia para el crecimiento económico coreano. Prioritariamente se han impulsado este tipo de acciones con sus aliados comerciales y geoestratégicos, con el fin de incrementar el capital humano, las capacidades tecnológicas y reforzar las alianzas. Europa, por su parte, no ha mostrado ser un destino del todo atractivo para los estudiantes coreanos y la estrategia pareciera no estar conduciendo precisamente a cambiar dicha situación, ni menos aún en el sentido inverso (estudiantes europeos en Corea). Por ello, esta vía de colaboración debiera ser uno de los focos de atención de los programas a implementar, si se desea fortalecer la relación en CyT entre la UE y Corea, pues a través de ella se generan interrelaciones que se transforman en los principales motores de las futuras actividades tecnológicas conjuntas.

En cuanto a la formación de extranjeros en Corea, la situación es diferente, pues esta nación recibe estudiantes fundamentalmente de China y de manera creciente, mientras que la formación de occidentales es muy reducida (gráfico 6). Como origen de estudiantes extranjeros que llegan cada año a Corea, el mayor número proviene de China, seguido de Japón y Estados Unidos, lo que está en concordancia con el destino de los Coreanos (OECD, 2013).

\section{GRÁFICO 6. ESTUDIANTES EXTRANJEROS DE EDUCACIÓN TERCIARIA EN UNIVERSIDADES COREANAS. INCLUYE ESTUDIANTES NO RESIDENTES Y NO CIUDADANOS COREANOS}

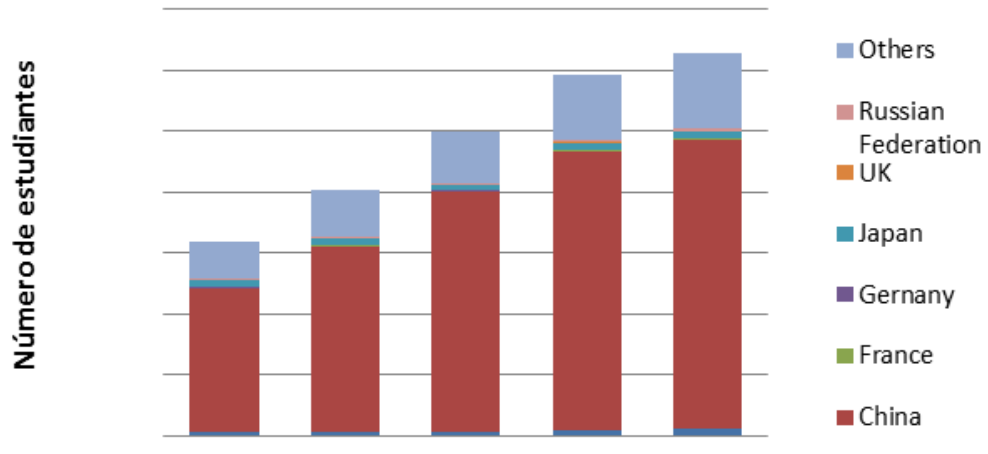

Fuente: Elaboración propia a partir de datos de la OECD, 2013

Las dificultades idiomáticas, lejanía geográfica, diferencias socioculturales, y las amplias oportunidades en occidente para sus estudiantes, podrían explicar la baja llegada a las universidades coreanas. Por su parte, Corea ofrece un atractivo espacio y una puerta a nuevas tecnologías para estudiantes chinos, lo que hace este destino sumamente llamativo.

Lo anterior muestra que este tipo de construcción de redes mantienen a Corea cerca de Occidente, conservando la hegemonía quienes han sido sus aliados en la historia reciente. A pesar de lo anterior, Japón sigue siendo también un socio estratégico importante en este tipo de iniciativas, lo que se ve favorecido por la cercanía geográfica, cultural y tecnológica. Por su parte, el impacto del gran incremento de estudiantes chinos en Corea podría marcar un cambio en la tendencia de este país como socio estratégico y tecnológico de occidente. 


\subsection{Investigación y desarrollo UE-Corea}

Si observamos en términos amplios la colaboración científica y tecnológica de Corea con socios internacionales a través de las patentes, indicador habitualmente empleado para representar los resultados de carácter tecnológico del proceso de investigación científica y tecnológica, podemos observar que existe poca cultura de trabajo colaborativo internacional, pues no más allá del 5 por 100 de las invenciones coreanas han sido desarrolladas en conjunto con inventores extranjeros y más aún, esta situación tiende a empeorar a lo largo del período analizado (1999 - 2010) para el caso de las patentes concedidas por la Oficina de patentes y marcas de los Estados Unidos (USPTO) ${ }^{2}$ (gráfico 7).

\section{GRÁFICO 7. PATENTES COREANAS CONCEDIDAS POR LA USPTO ENTRE 1999 Y 2010}

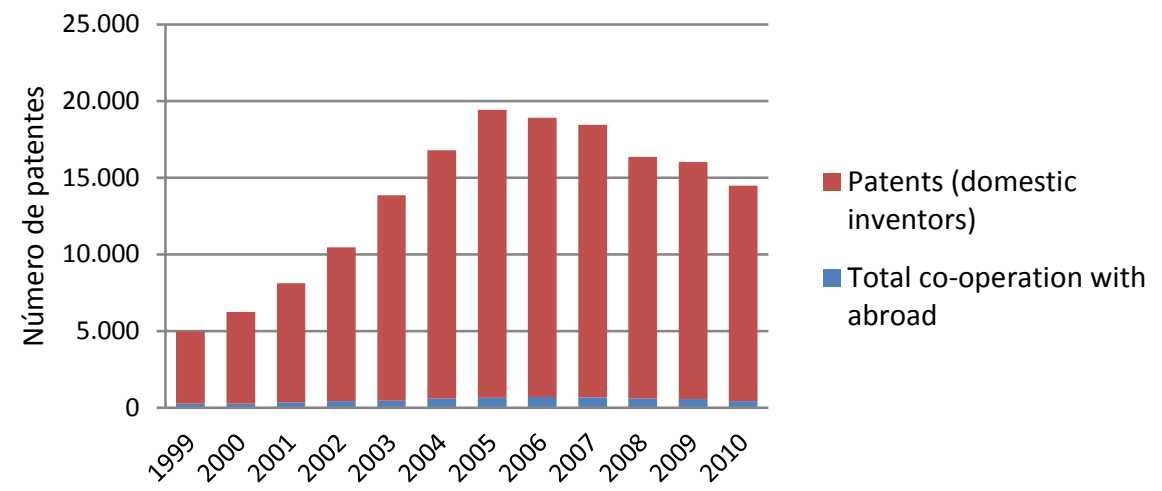

Fuente: Elaboración propia a partir de datos de la OECD, 2013

En término de origen de los coinventores, y según los datos de la OECD para las patentes concedidas por la USPTO, nuevamente encontramos a Estados Unidos como el más importante socio de Corea, quien representa casi el 50 por 100 del total de coinventores extranjeros (gráfico 8). Muy por detrás está la Unión Europea, la cual alcanza en promedio un 13 por 100 de participación, datos que son muy coincidentes con los encontrados en la educación terciaria. Japón, es el tercer país colaborador en el desarrollo de patentes con Corea, lo cual está asociado a los campos técnicos de la actividad conjunta empresarial.

En cuanto a la producción conjunta de conocimiento, expresado como co-publicaciones de artículos científicos, éste sigue el mismo patrón que las patentes, donde más del 50 por 100 de los artículos en coautoría internacional son con socios estadounidenses, mientras que Japón ocupa el segundo lugar y más atrás está Europa, particularmente Francia, Alemania y el Reino Unido. El cuarto socio en esta actividad científica corresponde a China (gráfico 9). Estos resultados son también coincidentes con los datos de formación en universidades extranjeras, lo que deja en evidencia lo interrelacionado que están todo este tipo de actividades y lo importante que es establecer estrategias equilibradas que contemplen las áreas de formación/educación, movilidad, I+D y desarrollos industriales.

\footnotetext{
${ }^{2}$ Esta situación sigue un patrón similar cuando se toma como referencia las patentes concedidas por la Oficina Europea de Patentes (EPO).
} 


\section{GRÁFICO 8. COINVENTORES EXTRANJEROS EN PATENTES COREANAS CONCEDIDAS POR LA USPTO 1999 - 2010}

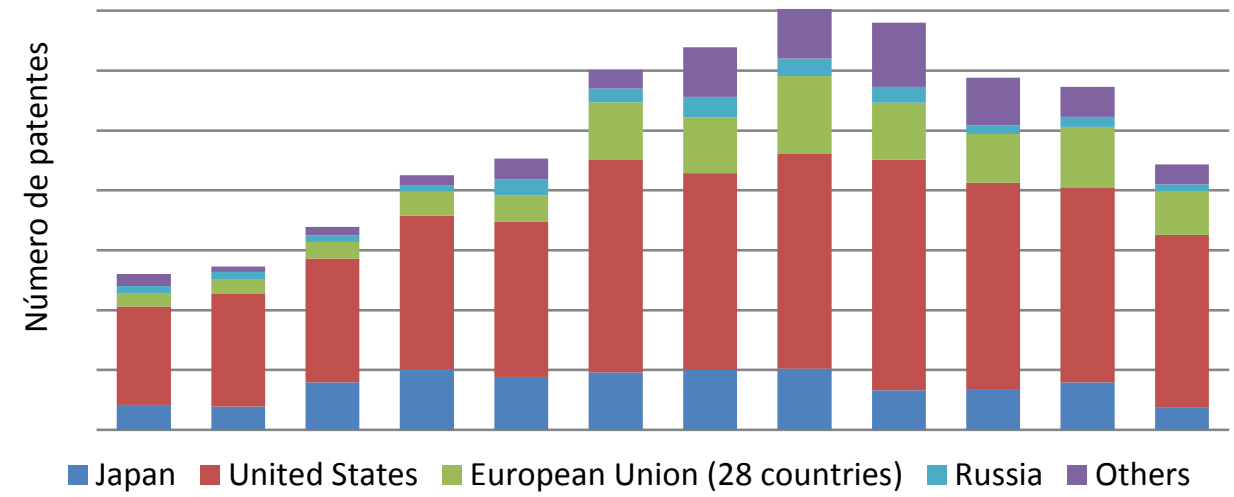

Fuente: Elaboración propia a partir de datos de la OECD, 2013

\section{GRÁFICO 9. CO-PUBLICACIONES CIENTÍFICAS COREANAS, POR ORIGEN DE COAUTOR.}

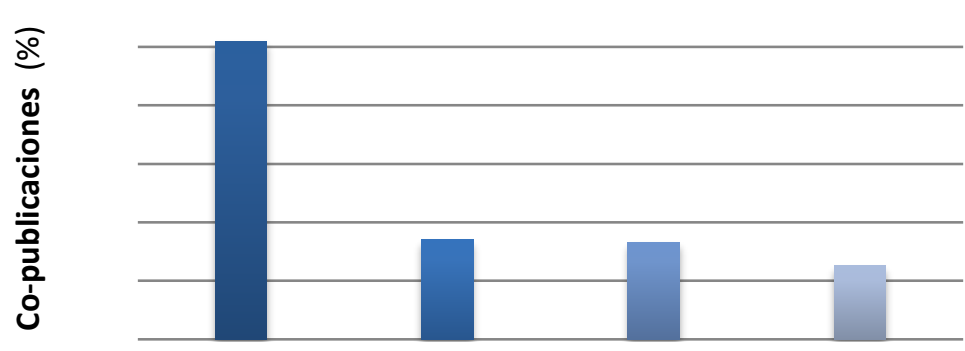
2010).

Fuente: Elaboración propia a partir de datos del Observatoire des Sciences et Techniques (Benaïm et al.,

Datos año 2008. EU(3) incluye Francia, Alemania y Reino Unido

Otra línea de trabajo donde se materializa la colaboración en CyT, es a través de los proyectos de $\mathrm{I}+\mathrm{D}$ entre equipos de investigadores de diferentes países. En efecto, las actividades de investigación conjunta son una vía habitual y creciente de colaboración, cuyos desafíos y objetivos son establecidos por quienes financian dichas acciones, los cuales también deciden el objeto y la conformación de la red de trabajo. Por ello, la unificación de las políticas de estado con los intereses industriales es esencial para el éxito de este tipo de políticas de colaboración.

Además de estas iniciativas cofinanciadas por el sector público, las empresas privadas emprenden directamente acciones internacionales de I+D con socios privados en el país de destino. Al respecto, las estadísticas ofrecen poca información sobre este tipo de colaboraciones, las que pueden ser entendidas como parte de la actividad empresarial de las firmas más innovadoras. Claro ejemplo de ello son las actividades de I+D que realizan las empresas multinacionales a través de sus filiales en el país de destino, generando flujos 
internos de tecnologías y conocimientos, mecanismo de creciente importancia (Keller, 2004; Lee, 2006).

En este sentido, uno de los indicadores que ofrece información al respecto es la Inversión Directa Extranjera (IDE), pues se reconoce que este tipo de actividad empresarial da origen a flujos de conocimientos y tecnologías entre el país huésped y la casa matriz, así como también en forma inversa. Las cifras entregadas por la OECD (2013) muestran que en los últimos años Japón, EUA y la UE (25) son los principales inversores en Corea, con aproximadamente el mismo nivel de flujos de capital (gráfico 10). Esto indicaría que a diferencia de los intercambios de conocimientos vía formación de alumnos o patentes, la UE posee una importancia relativa mayor en cuanto a colaboración tecnológica vía IDE, aunque Japón y EUA siguen siendo importantes socios de Corea.

\section{GRÁFICO 10. INVERSIÓN DIRECTA EXTRANJERA EN COREA}

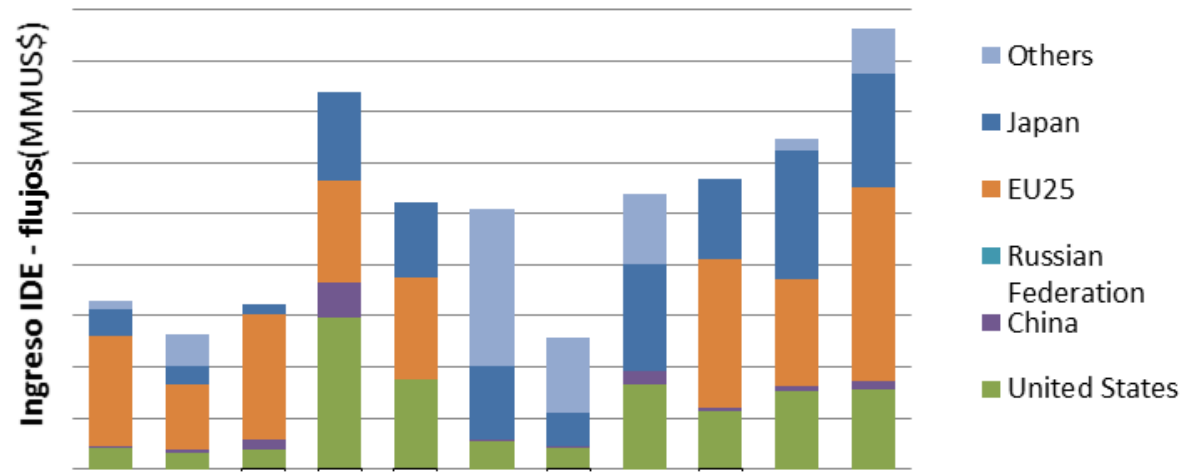

Fuente: Elaboración propia a partir de datos de la OECD (2013)

El valor total de la Inversión Directa Extranjera en Corea (gráfico 11) ha aumentado considerablemente en la última década, como lo muestras las cifras reportadas por la OECD (OECD, 2014). Está claro que este país es un destino atractivo para la inversión, fundamentalmente en aquellas áreas en las cuales presenta ventajas competitivas, como por ejemplo en las TICs (CNUCED, 2015).

Por su parte, la inversión extranjera de Corea tiene como principales destino la UE, EUA y China (gráfico 12), lo que demuestra la importancia de la UE en actividades desarrolladas a través del sector privado, así como también la relevancia que va tomando China como destino de deslocalización de empresas en la búsqueda de incremento en la competitividad, lo que no es opuesto al intercambio tecnológico, sino más bien es parte de una estrategia diseñada para tal propósito en el pasado (Hong et al., 2005).

En cuanto a la Inversión Directa extranjera total de Corea (gráfico 13), ésta ha incrementado aún más que el ingreso y las cifras totales son incluso superiores a este (OECD, 2014), lo que demuestra la habilidad de las empresas coreanas para incursionar en mercados externos vía localización, lo que favorece los flujos de tecnologías desde países más desarrollados, siguiendo lo propuesto por Cuervo-Cazurra (2007). 


\section{GRÁFICO 11. INVERSIÓN DIRECTA EXTRANJERA EN COREA. STOCK}

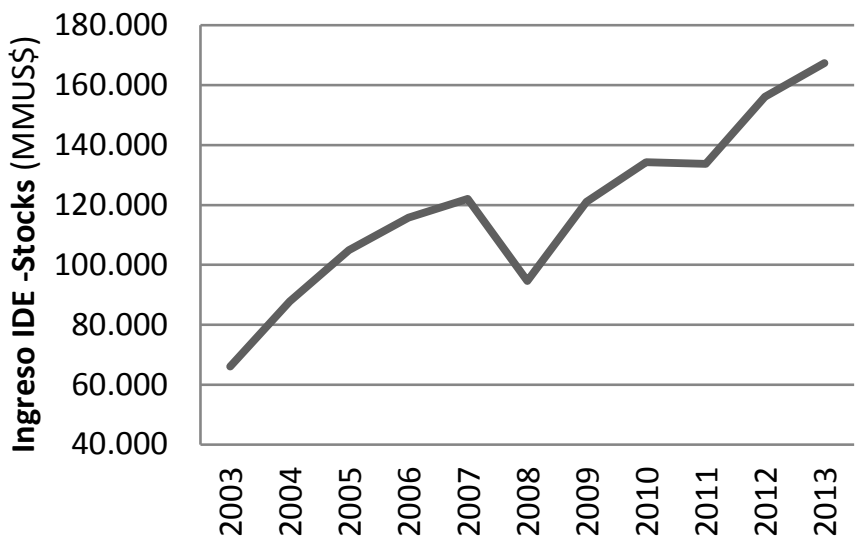

Fuente: Elaboración propia a partir de datos de la OECD (2014)

\section{GRÁFICO 12. INVERSIÓN DIRECTA EXTRANJERA COREANA. PRINCIPALES DESTINOS}

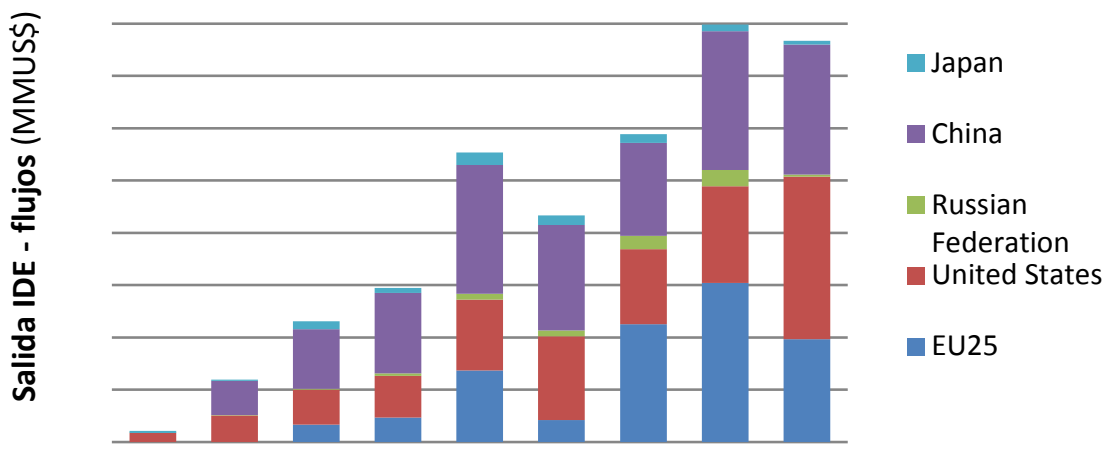

Fuente: Elaboración propia a partir de datos de la OECD (2013)

\section{GRÁFICO 13. INVERSIÓN DIRECTA EXTRANJERA COREANA. STOCK}

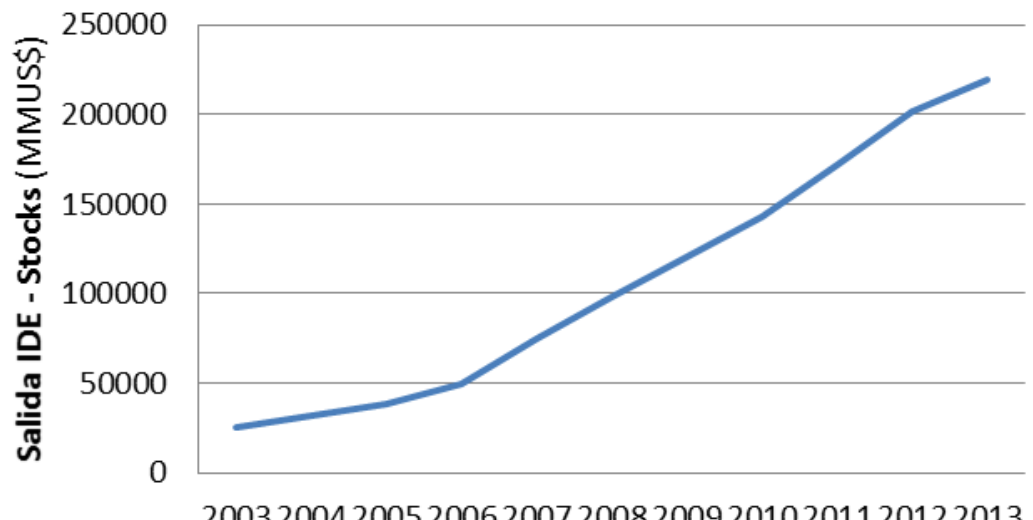

20032004200520062007200820092010201120122013

Fuente: Elaboración propia a partir de datos de la OECD (2014) 


\subsection{Colaboración internacional en CyT de Corea. Una mirada hacia la UE}

En términos oficiales, la colaboración en CyT entre la UE y Corea está enmarcada dentro del acuerdo firmado para tal efecto que entró en vigencia en abril de 2007. Este marco que ha sido recientemente actualizado ha entrado en vigor en julio de 2011 (European Commision, 2013a). Además de este acuerdo, Corea tiene diversos convenios bilaterales con la gran mayoría de los actuales países que conforman la UE, muchos de ellos anteriores a la década de los 80, cuando Corea aún estaba en sus fases iniciales de desarrollo. Unido a todo esto, está el tratado European Atomic Energy Community (EURATOM) UE-Corea, programa que ya tiene más de una década y que se ha traducido en la participación coreana en el proyecto de Fusión Atómica (ITER) y otras instancias relacionadas a temas nucleares liderados por Europa.

En particular, el Convenio marco de cooperación científica y tecnológica entre la Comunidad Europea y la República de Corea tiene su foco en tres áreas temáticas definidas de común acuerdo entre las partes y dos acciones de carácter transversal (European Commision, 2013a), que corresponden a:

- Nano ciencias, materiales y producción: nano-seguridad, manufactura y materiales críticos.

- Tecnologías de la información y comunicación: tecnologías futuras y emergentes, internet del futuro, robótica.

- Energía (no nuclear): fuentes energéticas alternativas, límite a la dependencia de combustibles fósiles, reducción de las emisiones de $\mathrm{CO}_{2}$ y mejoramiento de la eficiencia energética.

- Movilidad de investigadores

- Cooperación internacional

Los avances hasta la fecha no han sido muy destacados, tal como lo reflejan los informes emitidos desde la UE (European Commission, 2013a). En la práctica, se ha limitado a acciones orientadas a establecer agendas, redes de trabajo y algunas iniciativas científicas. Lo reducido de estos avances no es de sorprender, ya que por una parte, este acuerdo es reciente y por otra, la colaboración internacional coreana con Europa en CyT históricamente ha sido más bien limitada.

A pesar de lo anterior, la participación en iniciativas de I+D ha ido progresando. Ya en el Sexto Programa Marco (PM) 2002-2006, 18 iniciativas se pusieron en marcha con la colaboración coreana, mientras que en el Séptimo PM (2007-2013) este número ascendió a los $57^{3}$ (European Commission, 2013b). Unido a ello, se movilizaron diversas iniciativas con tendencia a fortalecer la construcción de redes de trabajo, bajo la orientación de las políticas de CyT europeas y coreanas, dando lugar a KORRIDOR (Stimulating and facilitating the participation of European researchers in Korean R\&D programmes), KESTCAP (Korea-EU Science and Technology Cooperation Advancement Programme), y más recientemente a KORANET (Korean Scientific Cooperation Network with the European Research Area) (cuadro 3).

\footnotetext{
${ }^{3}$ Proyectos contabilizados hasta fines del 2012
} 


\section{CUADRO 3. PRINCIPALES CARACTERÍSTICAS DE LOS PROGRAMAS DE COLABORACIÓN COREA-UE KORRIDOR, KESTCAP Y KORANET}

\begin{tabular}{cl}
\hline Programa & \multicolumn{1}{c}{ Características } \\
\hline KORRIDOR & $\begin{array}{l}\text { Proyecto desarrollado con el objetivo de ampliar y fortalecer la colaboración entre la UE y } \\
\text { Corea en áreas de común interés, a través de la apertura de oportunidades para logar una } \\
\text { mayor la participación de Corea en la I+D conjunta }{ }^{4} \text {. }\end{array}$ \\
KESTCAP & $\begin{array}{l}\text { Plataforma para fortalecer el acuerdo entre la UE y Corea en CyT, identificando campos de } \\
\text { común interés, contribuyendo al diálogo político en ciencia, tecnología e innovación. } \\
\text { KORANET }\end{array}$ \\
$\begin{array}{l}\text { Con el objetivo de fortalecer la red de trabajo científico entre la UE y Corea, esta iniciativa } \\
\text { se enfocó en las ya existentes redes de trabajo, y ha sido capaz de producir diversos } \\
\text { estudios conjuntos }{ }^{5} \text {. }\end{array}$ \\
\hline Fuente: Elaboración propia.
\end{tabular}

Los principales avances alcanzados como parte del Acuerdo de colaboración Corea-UE en CyT son:

\section{a. Energía}

A través de la iniciativa SETPLAN, se trabaja para ampliar la diversidad de fuentes de energía, reducir las emisiones de $\mathrm{CO}_{2}$ y limitar la dependencia de combustibles fósiles. A nivel estratégico este tema es de gran relevancia para ambas partes, pues Corea ha declarado en su estrategia Green Growth, la convicción por un desarrollo ambientalmente sostenible, mientras que para la UE y su Estrategia 2020, el cuidado del medioambiente es considerado una piedra angular del desarrollo.

\section{b. Nano-ciencias, Materiales y Producción}

Los principales avances quedan reflejados en los workshops llevados a cabo. En cuanto a materias, se busca el intercambio de conocimientos a través de la participación en eventos de esta naturaleza, además del diálogo político.

\section{c. Tecnologías de la información y comunicación}

Además de los proyectos impulsados en esta temática, Corea fue invitada al Experts Advisory Group, un claro ejemplo del deseo expreso de la UE en estrechar lazos en esta área.

\section{OPORTUNIDADES PARA LA COLABORACIÓN COREA-UE EN CIENCIA Y TECNOLOGÍA}

Para analizar las oportunidades de colaboración entre la UE y Corea, debemos poner la atención en las fortalezas y debilidades que tiene cada socio, así como en las estrategias definidas por cada parte. Al respecto, son bien conocidas las enormes capacidades humanas y tecnológicas con que cuenta la UE, en las diversas disciplinas científicas, destacando sus grandes y avanzadas infraestructuras científicas, un capital humano altamente especializado y un sistema de I+D moderno, interconectado y líder en muchas áreas. En cuanto a su estrategia, ésta se encuentra claramente definida por parte de la Comisión Europea y sus grupos afines, la cual puede ser observada ampliamente en la serie de documentos que emanan tanto del nivel europeo, como de sus Estados miembros. Dentro de los documentos estratégicos a destacar está la Estrategia 2020 y todos aquellos referidos al programa Horizonte 2020, el cual recoge las iniciativas que se encuentran en ejecución y aquellas que

\footnotetext{
${ }^{4}$ Más información puede ser obtenida en www.access4.eu/southkorea/626.php

${ }^{5}$ Más información puede ser obtenida en www.koranet.eu/
} 
serán llevadas a cabo como parte de las tareas que la UE ha fijado para los próximos años. Cabe destacar que las principales acciones a desarrollar, se enmarcan dentro de los siguientes puntos:

\section{- Ciencias Excelente}

- Tecnologías emergentes y futuras

- Liderazgo industrial

- En industrias tecnológicas

- Nanotecnologías, materiales avanzados, Proceso y manufactura avanzada, Biotecnología

- Espacio

- Innovación en PYMEs

- Retos sociales

- Salud, retos demográficos y bienestar

- Seguridad alimentaria, sustentabilidad de la agricultura y silvicultura, agua y bioeconomía

- Energía segura, eficiente y limpia

- Transporte inteligente, verde e integrado

- Medioambiente, eficiencia de los recursos y materias primas

- Sociedad inclusiva

- Seguridad

\section{- EURATON (energía nuclear)}

Haciendo un cruce entre las estrategias de Corea con las de la UE, podemos observar que existen importantes campos donde surgen oportunidades, más allá de aquellas áreas establecidas en el acuerdo firmado entre ambas partes. En efecto, el acuerdo de colaboración en temas científicos y tecnológicos establece una serie de temáticas que se encuentran priorizados tanto en la estrategia coreana como en la de la UE. Un detalle de las áreas de cooperación puede observarse en el cuadro siguiente (cuadro 4). La suma de las capacidades científicas, humanas e infraestructuras, son alicientes y una gran oportunidad para incrementar la asociación entre ambas partes, lo que se une a las decisiones estratégicas sobre mercados y productos que las empresas de ambas zonas están llevando a cabo.

Más allá de los temas priorizados en el acuerdo de colaboración, otras áreas también pueden ser objeto de trabajo colaborativo. Tal como se puede observar en el cuadro 4, salud o transporte podrían ser también temáticas atractivas. Del mismo modo, existe una tremenda oportunidad de impulsar acciones conjuntas en temas de carácter global, como es el caso del cambio climático.

De acuerdo a lo anterior, las principales y nuevas oportunidades se encuentran en las áreas de medioambiente, salud y sistema sanitario. Ambas áreas, de carácter global, están dentro de las agendas estratégicas de la UE y Corea, y pueden abordarse a nivel de grandes proyectos colaborativos, incluyendo a terceros países. Los temas en este ámbito podrían incluir tópicos tales como: cambio climático, residuos, transporte, energía, retos sanitarios, salud pública, nuevos patógenos, envejecimiento, alimentación, entre otros, donde tanto Corea como la UE tienen altas competencias, por lo que la búsqueda conjunta de soluciones puede ser de gran valor. Si bien es cierto que ya existen iniciativas conjuntas en estas áreas, 
éstas podrían reforzarse aprovechando la voluntad política para estrechar los lazos en CyT, brindando más y mejores resultados de índole tecnológica.

\section{CUADRO 4. PERSPECTIVA ESTRATÉGICA DE COREA, UE Y DEL ACUERDO DE COLABORACIÓN EN CIENCIA Y TECNOLOGÍA COREA-UE.}

\begin{tabular}{|c|c|c|c|}
\hline \multirow{2}{*}{ Temática } & \multicolumn{2}{|c|}{ Estrategia CyT } & \multirow{2}{*}{$\begin{array}{c}\text { Acuerdo colaboración } \\
\text { CyT } \\
\end{array}$} \\
\hline & Corea & UE & \\
\hline \multicolumn{4}{|l|}{ Visión estratégica } \\
\hline \multicolumn{4}{|l|}{ CyT como pilar del desarrollo } \\
\hline \multicolumn{4}{|l|}{$\begin{array}{l}\text { Empresa como vía de generación de } \\
\text { riqueza y desarrollo }\end{array}$} \\
\hline \multicolumn{4}{|l|}{ Énfasis en PYMEs } \\
\hline \multicolumn{4}{|l|}{$\begin{array}{l}\text { Incremento gasto en I+D para intensificar } \\
\text { el proceso innovador }\end{array}$} \\
\hline \multicolumn{4}{|l|}{ Áreas } \\
\hline Nuevas tecnologías & $\checkmark$ & $\checkmark$ & $\checkmark$ \\
\hline Salud/sanidad & $\checkmark$ & $\checkmark$ & \\
\hline TICs & $\checkmark$ & $\checkmark$ & $\checkmark$ \\
\hline Medio ambiente & $\checkmark$ & $\checkmark$ & \\
\hline Energía & $\checkmark$ & $\checkmark$ & $\checkmark$ \\
\hline Materiales & & $\checkmark$ & $\checkmark$ \\
\hline Alimentación & & $\checkmark$ & \\
\hline Seguridad & & $\checkmark$ & \\
\hline Transporte & & $\checkmark$ & \\
\hline Espacio & & $\checkmark$ & \\
\hline Acciones estratégicas & & & \\
\hline Proyectos de I+D & $\checkmark$ & $\checkmark$ & $\checkmark$ \\
\hline Movilidad & $\checkmark$ & $\checkmark$ & $\checkmark$ \\
\hline Educación & $\checkmark$ & $\checkmark$ & $\checkmark$ \\
\hline Diálogo para conformar agendas & $\sqrt{ } 7$ & $\checkmark$ & $\checkmark$ \\
\hline Acciones en colaboración público-privado & $\checkmark$ & $\checkmark$ & \\
\hline
\end{tabular}

Producto del énfasis puesto en el sector privado como eje de las políticas de innovación para lograr un desarrollo sostenible enfocadas en las empresas, las acciones en programas tales como los del tipo public private partnerships projects son también una buena vía de trabajo colaborativo y a la vez altamente enfocada en resultados aplicables en la industria.

Además de las actividades de I+D posibles a desarrollar bajo la perspectiva de los programas diseñados por el Estado para dicho propósito, se encuentran las múltiples colaboraciones que se realizan directamente entre empresas privadas, sin la concurrencia de fondos públicos y que posiblemente pueden ser de mayor dimensión, al menos en término de número de iniciativas.

Por su parte, un área donde se observa una baja colaboración, y que es la base para el establecimiento de redes y futuras acciones conjuntas en $\mathrm{I}+\mathrm{D}$, es la formación de coreanos en

\footnotetext{
${ }^{6}$ Horizonte 2020 tiene un incremento relevante en el presupuesto, en comparación al $7^{\circ}$ Programa Marco

${ }^{7}$ No se indica expresamente como mecanismo de búsqueda de consensos.
} 
Europa y viceversa. Finalmente, es necesario hacer mención del efecto que significa el crecimiento de China, vecino de Corea y con históricos lazos socioculturales y económicos, sobre las oportunidades que ofrecerá a este país, lo que podría ser un importante competidor frente a los intereses europeos.

\section{CONCLUSIONES}

Corea ha realizado grandes y fructíferos esfuerzos para converger con las principales economías del mundo, lo que ha significado una profunda transformación industrial que la ha llevado a convertirse en líder mundial en diversas industrias de alta tecnología. Históricamente, el desarrollo industrial tuvo una fuerte base en tecnologías importadas, gracias a la capacidad de absorción desarrollada en Corea con un fuerte liderazgo estatal, además de un sistema bancario sano controlado por el estado y el predominio de los chaebol.

A raíz de la crisis financiera de 1997-1998, que azotó con fuerza a Corea, se revisó el modelo de desarrollo, con una importante reestructuración de los chaebol y un papel de liderazgo reducido para el Estado. La economía se recuperó rápidamente, hasta alcanzar un ingreso per cápita de alrededor de 70 por 100 de la media de la OCDE. Otro claro signo de progreso fue el aumento de las exportaciones de productos de media-alta y alta tecnología, debido al desarrollo de las capacidades tecnológicas en una serie de áreas, lo que luego estaría acompañado por la salida de sus empresas que se convertirían en importantes compañías multinacionales.

Este éxito tiene pocas similitudes en el mundo. Sin embargo, Corea está entrando en una nueva y crítica fase en su desarrollo y se enfrenta a nuevos retos. Más que nunca, mantener el crecimiento económico dependerá del impulso de la innovación, es por ello que la política de innovación de Corea busca acelerar el paso del "catch-up" a un sistema de innovación "creativo", pues el enfoque tradicional de convergencia asociado a la captura internacional de tecnologías no ha sido propicio para la creación de nuevas empresas innovadoras, la transferencia de tecnologías, o para la construcción de capacidades de investigación básica. Este nuevo modelo se basa en gran medida en el aumento del gasto en I+D, y en mejorar los flujos de conocimiento y transferencia de tecnologías en todo el sistema. En particular se busca incrementar la relevancia de las PYMEs, dar un mayor énfasis a la investigación fundamental, propiciar la participación activa de las universidades en las actividades de I+D, y propiciar la internacionalización del sistema de investigación nacional. Lo anterior está dando importantes frutos, toda vez que la IDE es cada vez más importante, nuevas empresas multinacionales entran al país y realizan $\mathrm{I}+\mathrm{D}$, así como las domésticas salen y capturan nuevo conocimiento, todo lo cual impulsa nuevas innovaciones.

Sin embargo, más allá de la actividad de las EMN, Corea ha mostrado una acción reducida en términos de colaboración internacional en CyT. Dentro de las principales actuaciones están la formación profesional de coreanos en el extranjero y la de extranjeros en Corea, mientras que las actividades de $\mathrm{I}+\mathrm{D}$ en colaboración con socios extranjeros son más recientes, destacando Japón y Estados Unidos como aliados, tanto a nivel de desarrollo tecnológico como en el destino de sus estudiantes, lo que se debe a sus lazos socioculturales y cercanía geográfica en el caso de Japón, y a la vinculación estratégica y económica en el caso de EUA. Por su parte China, ha ido tomando relevancia y se proyecta como un socio de relevancia en el futuro, lo que está asociado a su desarrollo económico y tecnológico, cercanía geográfica y sociocultural, lo cual representa un importante eje de atracción para la CyT de Corea. 
En este contexto, la UE aparece como un colaborador de baja importancia, a pesar de compartir una agenda de desarrollo, tener acuerdos establecidos en esta materia, y constituir una gran oportunidad para ambos. Hasta la fecha, Europa ha sido para Corea fundamentalmente un destino para sus estudiantes y un socio tecnológico de menor relevancia, situación que muestra algunos signos de transformación en los últimos años, como consecuencia del interés de los Estados de estrechar lazos y de las empresas multinacionales que buscan nuevas tecnologías y mercados.

Las oportunidades de incrementar la colaboración entre Corea y la UE son amplias, no sólo por el bajo nivel comparativo actual, sino también porque las agendas estratégicas de ambos socios son altamente coincidentes, incluso más allá de los términos que aborda el acuerdo de colaboración vigente. Áreas como la salud, medioambiente y tecnologías (nuevas $\mathrm{y}$ futuras) son espacios donde ambos bloques poseen enormes competencias y retos de carácter global.

Otras vías plausibles para acercar Corea y la UE es el despliegue de iniciativas con tendencia a promover las universidades europeas como destino de estudiantes coreanos, con el objeto de estrechar lazos, crear redes e incrementar la participación en proyecto de I+D.

Este trabajo tiene importantes implicaciones, tanto para Corea como la UE, pues caracteriza y cuantifica la colaboración, identificando las principales vías para incrementarla, haciendo una llamada de atención a los responsables de las políticas públicas para conducir este proceso y poner especial hincapié en direccionar la IDE y las EMN con el fin de estimular la I+D colaborativa y enfocada en dar respuesta a los desafíos conjuntos de estas economías. Por otra parte, este estudio muestra claramente la importancia de las relaciones socioculturales entre los países, como base para el desarrollo conjunto de actividad vinculada a la CyT, así como el efecto que pueden tener los acuerdos geoestratégicos para un país, como fue el caso de EUA y Corea luego de la Segunda Guerra Mundial, lo cual termina impactando los procesos endógenos de I+D.

Este estudio presenta limitaciones derivadas de la disponibilidad de datos y la multidimensionalidad de los procesos científicos y tecnológicos, así como de colaboración entre países. Por ello, a pesar de analizar las variables más comúnmente utilizadas en el estudio de la colaboración en CyT y revisar diversos estudios y documentos oficiales coreanos, es probable que una serie de aristas no hayan sido del todo descubiertas, abriendo matices al entendimiento de la colaboración que pudiesen reforzar aún más este trabajo. A pesar de ello, las conclusiones son coherentes con la teoría económica evolutiva y aborda los principales elementos que constituyen los pilares de los procesos de innovación, de acuerdo a lo establecido por organismos internacionales como la OECD, Unión Europea y el Banco Mundial.

\section{BIBLIOGRAFÍA}

Aizawam, M., Prasad, L., Su-Yeon, M. y Guoping, Z. (2007): Políticas de $I+D$ en Asia. Japón, India, China y Corea del Sur, Casa Asia editores, Barcelona, España.

Archibugi, D. y Michie, J. (1995): “The globalisation of technology: a new taxonomy”, Cambridge journal of Economics, 19(1), pp. 121-140.

Athreye, S. y Cantwell, J. (2007): "Creating competition?: Globalisation and the emergence of new technology producers”, Research Policy, 36(2), pp. 209-226. 
Benaïm, M., Laurens, P., Laville, F., Mérindol, V., Ramanana-Rahary, S., Roth, C., Taillibert, M. y Virlon, B. (2010): Indicateurs de sciences et de technologies, Edición 10, Economica \& OST, París.

Bouteiller, E. y M. Fouquin (2001): Le développement économique de l'Asie orientale, Collection Repères La Découverte, Paris.

Cuervo-Cazurra, A. (2007): "Sequence of value-added activities in the multinationalization of developing country firms”, Journal of international Management, 13(3), 258-277.

CNUCED (2015): Informe sobre las inversiones mundiales: 2015, Reforma de la gobernanza internacional en materia de inversiones, Naciones Unidas, Nueva York.

Eckert, C., Lee, K., Lew, Y., Robinson, M. y Wagner, E. (1990): Korea old and new: a history, Korean Institute, Harvard University.

European Commission (2013a): Second EU-Korea Cooperation Roadmap in Science, Technology and Innovation 2011-2013, disponible en http://ec.europa.eu/research/iscp/pdf/korea_roadmap_2011-2013.pdf, visitado el 28 de noviembre de 2013.

European Commission (2013b): Research \& Innovation. International Cooperation, disponible en http://ec.europa.eu/research/iscp/index.cfm?lg=en\&pg=korea, revisado el 29 de noviembre de 2013.

European Commision (2015) Republic of Korea, Country page. Disponible en ec.europa.eu/research/. Revisado el 15 de noviembre de 2016.

Foray, D. (2002): “La sociedad del conocimiento", Revistas Internacionales de Ciencias Sociales, $\mathrm{n}^{\mathrm{o}} 171$ Marzo 2002, UNESCO, disponible en http://www.unesco.org/new/fileadmin/MULTIMEDIA/HQ/SHS/pdf/171fulltext171spa.pdf.

Foreign and Commonwealth Office (2013): South Korea: New Basic Plan for Science and Technology, July 2013, disponible en http://opentoexport.com/article/south-koreanew-basic-plan-for-science-and-technology-july-2013/. Visitado el 1 de diciembre de 2013.

Furman, J., Porter, M., y Stern, S. (2002): “The determinants of national innovative capacity”, Research Policy, 31(6), pp. 899-933.

García-Blanch, F. (2002): Crecimiento económico en Corea del Sur (1961-2000): aspectos internos y factores internacionales, Síntesis, Madrid.

Henderson, J. (1993): "The Role of the State in the Economic Transformation of East Asia", en C. Dixon y D. Drakakis-Smith (editores), Economic and Social Development in Pacific Asia, Routledge, London.

Hong, S., Popper, S., and Zheng, K. (2005): Strategic Choices in Science and Technology. Korea in the Era of a Rising China, RAND Corporation.

Hong, S., Chanmi J. and Jonglip K. (2012): The Internalization of Science and Technology in the earlier stage of Economic Development in South Korea, Ministry of Strategy and Finance (MOSF), Republic of Korea.

ICEX (2013): ICEX España. Exportaciones inversiones, disponible en http://www.oficinascomerciales.es/icex/cda/controller/pageOfecomes/0,5310,5280449 _5304715_5296234_0_KR,00.html. Visitado el 03 de diciembre de 2013.

Keller, W. (2004): “International Technology Diffusion”, National Bureau of Economic Research, Centre for Economic Policy Research, abril. 
Lee, W. (2006) "Multinationals, Globalization, and Public Policy in the Republic of Korea towards FDI”, KOTRA International Conference, 14 october de 2006.

Lee, W. (1997): "The Role of Science and Technology Policy in Korea's Industrial Development”, Simposio internacional: Innovation and Competitiveness in Newly Industrializing Economies, Seoul, Korea, May 26-27, 1997.

Lim, Y. (2000): "Development of the Public Sector in the Korean Innovation System", International Journal of Technology Management, 20(5/6/7/8).

MEST (2008): Becoming an S\&T Power Nation through the 577 Initiative, Science and Technology Basic Plan of the Lee Myung Bak Administration, Ministry of Education, Science and Technology, Seoul.

MoST (2013): Minister of Science and Technology, disponible en http://park.org/Korea/Pavilions/PublicPavilions/Government/most/index.html, visitado el 27 de noviembre de 2013.

MoST y KISTEP (2004): Korea Institute of S\&T Evaluation and Planning 2004 Report on the Survey of Research and Development in Science and Technology, Ministry of Science and Technology Republic of Korea.

MoST y KISTEP (2006): Report on the Survey of Research and Development in Science and Technology, Ministry of Science and Technology and Korea Institute of Science and Technology Evaluation and Planning, Seoul (in Korean).

MoST y KISTEP (2007): Report on the Survey of Research and Development in Science and Technology, Ministry of Science and Technology and Korea Institute of Science and Technology Evaluation and Planning, Seoul.

OECD (1996): Reviews of National Science and Technology Policy - Republic of Korea.

OECD (2006): OECD Thematic Review of Tertiary Education, Country Background Report for Korea.

OECD (2007): PISA 2006: Science Competencies for Tomorrow's World, OECD, Paris.

OECD (2009a): OECD Economic Surveys - Korea, OECD, Paris.

OECD (2009b): OECD Reviews of Innovation Policy - Korea.

OECD (2013): OECD. Stat extracts, disponible en http://stats.oecd.org, visitado el 27 de noviembre de 2013.

OECD (2014): OECD. Stat extracts, disponible en http://stats.oecd.org, visitado el 14 de mayo de 2015.

OECD (2015): PISA 2015 Assessment and Analytical Framework, Science, reading mathematic and financial literacy, Pisa, OECD Publishing, Paris.

OECD (2016): Main Science and Technology Indicators Database, January 2016.

Park, B.M. (2004): “Major Development and Achievements of Korea's S\&T Policy 2003”, International Workshop on the Comprehensive Review of the Basic S\&T PlansSTEPI and TECHNOVALUE (2004), Strategic Direction for Government-sponsored Research Institutes, Science and Technology Policy Institute.

Suh, J. H. (2000): “Korea's Innovation System: Challenges and New Policy Agend”a, Discussion Paper Maastricht: Institute for New Technologies, $\mathrm{n}^{\circ}$ 2004-4.

The Republic of Korea (2015): The Republic of Korea. Cheong Wa Dae, Sitio web disponible en http://english1.president.go.kr. Revisado el 10 de abril de 2016. 
UNDP (2015): Informe sobre Desarrollo Humano 2015, Trabajo al servicio del desarrollo humano, Ediciones PBM Graphics, Nueva York.

UNESCO (2015): Institute for Statistics. Disponible en http://www.uis.unesco.org/Pages/default.aspx.

World Bank (2014): World Development Indicators. Disponible en http://data.worldbank.org. Revisado en 20 de junio de 2014

World Bank (2016): World Bank Open Data. Disponible en www.data.worldbank.org/. Revisado el 15 de noviembre de 2016.

\section{ANEXOS}

Anexo 1. Crecimiento del consumo, inversión y comercio exterior de Corea

\begin{tabular}{|c|c|c|c|c|c|c|c|c|c|}
\hline \multirow{2}{*}{\multicolumn{2}{|c|}{ PIB }} & $\begin{array}{l}\text { 1998-2002 } \\
\text { Promedi }\end{array}$ & $\begin{array}{l}\text { 2002-2006 } \\
\text { Anual }\end{array}$ & $\begin{array}{l}2008 \\
\text { Cambic }\end{array}$ & \multicolumn{4}{|c|}{ Cambios porcentuales, volumen (a precios del 2005) } & $5)$ \\
\hline & & 7.20 & 4.30 & 2.30 & 0.30 & 6.30 & 3.60 & 3.50 & 4.30 \\
\hline Consumo privado & & 8.20 & 1.50 & 1.30 & 0.00 & 4.40 & 2.30 & 2.10 & 3.80 \\
\hline Consumo público & & 3.80 & 4.30 & 4.30 & 5.60 & 2.90 & 2.10 & 2.70 & 3.00 \\
\hline Formación bruta de capital & & 6.70 & 2.70 & -1.90 & -1.00 & 5.80 & -1.10 & 2.10 & 4.60 \\
\hline Acumulación de activos & & $\cdots$ & $\cdot$ & 0.60 & -3.90 & 2.50 & 0.80 & 0.50 & 0.00 \\
\hline Demanda interna total & & 8.00 & 2.30 & 1.30 & -3.40 & 7.20 & 2.00 & 2.70 & 3.80 \\
\hline $\begin{array}{l}\text { Exportación de bienes } \\
\text { servicios }\end{array}$ & & 10.80 & 14.10 & 6.60 & -1.20 & 14.70 & 9.50 & 5.10 & 9.80 \\
\hline $\begin{array}{l}\text { Importación de bienes } \\
\text { servicios }\end{array}$ & $\mathrm{y}$ & 12.60 & 13.10 & 4.40 & -8.00 & 17.30 & 6.50 & 3.50 & 9.00 \\
\hline Exportaciones netas & & & & 1.00 & 3.70 & -0.60 & 1.80 & 1.00 & 0.60 \\
\hline
\end{tabular}

1. Datos históricos.

2. Sobre la base de las proyecciones trimestrales producidos por OECD Economic Outlook, No 90.

3. Las contribuciones a los cambios en el PIB real (porcentaje del PIB real en el año anterior).

Fuente OECD (2014) 